\title{
The Moduli Space Metric for Well-Separated Non-Abelian Vortices
}

\author{
Toshiaki Fujimori ${ }^{1}$, Giacomo Marmorini², Muneto Nitta ${ }^{2}$, \\ Keisuke Ohashi ${ }^{3}$ and Norisuke Sakai ${ }^{4 *}$ \\ ${ }^{1}$ Department of Physics, Tokyo Institute of Technology, Tokyo 152-8551, Japan \\ 2 Department of Physics, and Research and Education Center for Natural Sciences, \\ Keio University, Hiyoshi 4-1-1, Yokohama, Kanagawa 223-8521, Japan \\ 3 Department of Physics, Kyoto University, Kyoto 606-8502, Japan \\ 4 Department of Mathematics, Tokyo Woman's Christian University, Tokyo 167-8585, Japan
}

October 22, 2018

\begin{abstract}
The moduli space metric and its Kähler potential for well-separated non-Abelian vortices are obtained in $U(N)$ gauge theories with $N$ Higgs fields in the fundamental representation.
\end{abstract}

${ }^{*}$ Email addresses: fujimori(at)th.phys.titech.ac.jp, giacomo(at)phys-h.keio.ac.jp, nitta(at)phys-h.keio.ac.jp, ohashi(at)gauge.scphys.kyoto-u.ac.jp, sakai(at)lab.twcu.ac.jp 


\section{Introduction}

Solitons, namely smooth localized solutions of nonlinear partial differential equations, have a long history in mathematical and physical sciences and can now be considered as a subject on their own. The number of physically relevant applications of soliton theory is huge and ranges from nonlinear optics to astrophysics. While in the mathematical literature the term soliton is mostly associated to integrable systems, in the framework of modern Lorentz-invariant field theories it refers to smooth localized solutions of field equations, that in general do not exhibit integrability. A particularly interesting class of solitons is represented by those solutions of the field equations which satisfy Bogomolnyi-Prasad-Sommerfield (BPS) bound, that is a lower bound for the energy functional. They are topologically stable and can be shown to actually satisfy first order, instead of second order, partial differential equations that do not involve time derivatives (BPS equation). Abrikosov-Nielsen-Olesen (ANO) vortices [1] at critical coupling , 't Hooft-Polyakov monopoles with massless Higgs field [2] and instantons in Euclidean Yang-Mills theory [3] are prominent examples (a standard reference is [4]). One characteristic feature of BPS solitons is that there exist no static forces among them. Therefore a large number of soliton configurations are allowed with degenerate energy, and consequently generic solutions contain moduli parameters (collective coordinates). The space of solutions of a given set of BPS equations is called moduli space and is parameterized by those moduli parameters.

The complete characterization of soliton moduli space is not only mathematically attractive, but has deep physical implications. In fact, while the dynamics of solitons in the full field theory is usually inaccessible (sometimes even numerically), following the idea of Manton [5] one can argue that at sufficiently low energies the time evolution is constrained by potential energy to keep the field configuration close to the moduli space, which is in general finite dimensional. The problem is then reduced to analyze the motion on the moduli space, which is actually a geodesic motion of the metric induced by the kinetic term of the field theory Lagrangian.

However, although a number of mathematical structures have been found in various cases, the explicit determination of moduli space metric can be very difficult in practice. For example, the moduli space of $k S U(2)$ monopoles reveals a hyper-Kähler structure, which is quite restrictive, but the metric is explicitly known only in the $k=2$ case, namely the Atiyah-Hitchin metric [6, 7]. Even in this case the geodesic motion is not integrable, except in a situation where one is allowed to use the asymptotic form of the metric. The asymptotic metric for well-separated BPS monopoles was constructed by Gibbons and Manton [8]. For other gauge groups the WeinbergLee-Yi metric is well known [9].

For BPS Abelian vortices (ANO vortices) in flat space $\mathbb{R}^{2}=\mathbb{C}$, the $k$-vortex moduli space was shown to be Kähler and a symmetric product $\mathcal{M}_{k} \simeq \mathbb{C}^{k} / \mathcal{S}_{k}$, where $\mathcal{S}_{k}$ denotes symmetrization [10]. For the metric on it, a major step was made in the work of Samols [11, where a general formula for the metric was given in terms of local data of the solutions of BPS equations. A Kähler potential for such metric could then be found easily (for a direct approach to the calculation of the Kähler potential with different arguments see [12]). Subsequently Manton and Speight [13] calculated the local data for well-separated vortices and, making use of Samols' formula, explicitly 
wrote down the asymptotic expression of the moduli space metric for $k$ vortices. Recently the moduli space metric was given for vortices on a hyperbolic space [14] in which case the system is integrable [15].

BPS non-Abelian vortices in more general Higgs models with non-Abelian gauge symmetry were introduced in [16, 17] (for a review see [18, 19]). Such configurations are parametrized not only by position moduli, but also by orientational moduli, that appear due to the presence of a non-trivial internal color-flavor space; it was found that a single vortex moduli space is

$$
\mathcal{M}_{k=1, N} \simeq \mathbb{C} \times \mathbb{C} P^{N-1}
$$

for $U(N)$ gauge theory with $N$ Higgs fields in the fundamental representation. The Kähler class on $\mathbb{C} P^{N-1}$ was determined to be $4 \pi / g^{2}$, with $g$ the gauge coupling constant [20]. The analysis of the moduli space has gone through many developments especially after the introduction of the moduli matrix formalism [21]-[30] (for a review of the method see [19]). The moduli matrix is a matrix whose components are holomorphic polynomials of $z$ (codimensions of vortices), and it contains all moduli parameters in coefficients [21]. The moduli space of multiple vortices at arbitrary positions with arbitrary orientations in the internal space was constructed in [24]. A general formula for the Kähler potential on the moduli space was obtained in [25]. For separated (not necessary well-separated) non-Abelian vortices, the moduli space can be written as the symmetric product of $k$ copies of the single vortex moduli space (1.1) [24]:

$$
\mathcal{M}_{k, N} \leftarrow\left(\mathbb{C} \times \mathbb{C} P^{N-1}\right)^{k} / \mathcal{S}_{k}
$$

where the arrow denotes the resolution of sigularities; The space on the right hand side contains orbifold singularities which correspond to coincident vortices, while the full moduli space $\mathcal{M}_{k, N}$ on the left hand side should be regular. By evaluating the Kähler potential [25] of the moduli space at linear order, it was explicitly shown in [27] that the metric is actually regular everywhere even at coincident limits of two vortices [26]. The head-on-collision of two vortices was also studied in [27].

The purpose of the present paper is to give the metric and its Kähler potential on the moduli space (1.2) for well-separated non-Abelian vortices. Our main results are the generalization of Samols' formula to the non-Abelian case and, starting from that and from the asymptotics of nonAbelian vortex solutions [30], the derivation of the explicit metric and its Kähler potential. The final form of the metric exhibits an evident interplay between spatial (position) and orientational moduli, opening up a rich variety of possibly interesting dynamics, that will be the object of a further investigation [31. In this paper we concentrate on local vortices, namely vortices in $U(N)$ gauge theories with Higgs fields in the fundamental representation in the same number as the number $N$ of colors (while semi-local vortices [32] exist in theories with more fundamental Higgs fields [33, 29]). We also restrict ourselves to $U(N)$ gauge group although non-Abelian vortices with gauge group $G \times U(1)$ with arbitrary simple group $G$ have been recently constructed in [34]-[38]. We leave generalizations to those cases as future works.

The paper is organized as follows. In Section 2 we define the model and review the construction of non-Abelian vortices in the moduli matrix formalism. In Section 3 we find the non-Abelian 
extension of the Samols' formula for the metric on the moduli space and in Section 4 we show how it can be made explicit in the case of well-separated vortices, which means to find the asymptotic metric and its Kähler potential. In Section 5 we obtain the latter result by means of a more physical method, namely point-particle approximation. Some details of the calculation are given in Appendix A.

\section{Review of non-Abelian local vortices}

\subsection{Lagrangian and BPS equations}

Let us consider a $U(N)$ gauge theory in $(2+1)$-dimensional spacetime with gauge fields $w_{\mu}$ for $U(1), W_{\mu}^{a}\left(a=1, \ldots, N^{2}-1\right)$ for $S U(N)_{C}$ and $N$ Higgs fields $H^{A}(A=1, \ldots, N)$ in the fundamental representation of the $S U(N)_{C}$ gauge group. The Lagrangian of the theory takes the form

$$
\begin{aligned}
\mathcal{L} & =-\frac{1}{4 e^{2}}\left(f_{\mu \nu}\right)^{2}-\frac{1}{4 g^{2}}\left(F_{\mu \nu}^{a}\right)^{2}+\left(\mathcal{D}^{\mu} H^{A}\right)^{\dagger} \mathcal{D}_{\mu} H^{A}-V, \\
V & =\frac{e^{2}}{2}\left(H_{A}^{\dagger} t^{0} H^{A}-\xi\right)^{2}+\frac{g^{2}}{2}\left(H_{A}^{\dagger} t^{a} H^{A}\right)^{2}
\end{aligned}
$$

where $\xi$ is the Fayet-Iliopoulos parameter, $e$ and $g$ are gauge coupling constants for $U(1)$ and $S U(N)_{C}$, respectively. Our notation is $\mathcal{D}_{\mu} H^{A}=\left(\partial_{\mu}+i w_{\mu} t^{0}+i W_{\mu}^{a} t^{a}\right) H^{A}$ and $f_{\mu \nu} t^{0}+F_{\mu \nu}^{a} t^{a}=$ $-i\left[\mathcal{D}_{\mu}, \mathcal{D}_{\nu}\right]$. The matrices $t^{0}$ and $t^{a}$ are the generators of $U(1)$ and $S U(N)$, normalized as

$$
t^{0}=\frac{1}{\sqrt{2 N}} \mathbf{1}_{N}, \quad \operatorname{Tr}\left(t^{a} t^{b}\right)=\frac{1}{2} \delta^{a b}
$$

As is well known, the Lagrangian Eq. (2.1) can be embedded into a supersymmetric theory with eight supercharges. The Higgs fields can also be expressed as an $N$-by- $N$ matrix on which the $S U(N)_{C}$ gauge transformations act from the left and the $S U(N)_{F}$ flavor symmetry acts from the right

$$
H \rightarrow U_{C} H U_{F}^{\dagger}, \quad U_{C} \in S U(N)_{C}, \quad U_{F} \in S U(N)_{F}
$$

Using this matrix notation for the Higgs fields, the vacuum condition can be written as

$$
H H^{\dagger}-v^{2} \mathbf{1}_{N}=0, \quad v^{2} \equiv \sqrt{\frac{2}{N}} \xi .
$$

The vacuum of this model is in an $S U(N)_{C+F}$ color-flavor locking phase, where the vacuum expectation values (VEVs) of the Higgs fields are

$$
H=v \mathbf{1}_{N}
$$

In this vacuum, the mass spectrum is classified according to the representation of $S U(N)_{C+F}$

$$
m_{e}=e v, \quad m_{g}=g v,
$$


where $m_{e}$ is for singlet fields and $m_{g}$ is for adjoint fields.

Considering a static configuration, the BPS bound for the energy reads

$$
E \geq-v^{2} \int d^{2} x \operatorname{Tr}\left(f_{12} t^{0}\right)=2 \pi v^{2} k, \quad k \in \mathbb{Z}
$$

The bound is saturated if the following BPS equations are satisfied:

$$
\mathcal{D}_{\bar{z}} H=0, \quad \frac{2}{e^{2}} f_{12} t^{0}+\frac{2}{g^{2}} F_{12}^{a} t^{a}=H H^{\dagger}-v^{2} \mathbf{1}_{N}
$$

where $z=x^{1}+i x^{2}$ is a complex coordinate. To solve the BPS equations, it is convenient to rewrite the gauge fields in terms of matrices $S_{e}\left(\propto \mathbf{1}_{N}\right) \in \mathbb{C}^{*}$ and $S_{g} \in S L(N, \mathbb{C})$

$$
\begin{aligned}
w_{\bar{z}} t^{0} & \equiv \frac{1}{2}\left(w_{1}+i w_{2}\right) t^{0}=-i S_{e}^{-1} \bar{\partial} S_{e} \\
W_{\bar{z}}^{a} t^{a} & \equiv \frac{1}{2}\left(W_{1}^{a}+i W_{2}^{a}\right) t^{a}=-i S_{g}^{-1} \bar{\partial} S_{g} .
\end{aligned}
$$

Then, the first BPS equation $\mathcal{D}_{\bar{z}} H=0$ can be solved as

$$
H=S_{e}^{-1} S_{g}^{-1} H_{0}(z)
$$

where $H_{0}(z)$ is an arbitrary $N$-by- $N$ matrix which is holomorphic in $z$. The second BPS equation becomes [21, 24]

$$
\frac{4}{m_{e}^{2}} \bar{\partial}\left(\Omega_{e} \partial \Omega_{e}^{-1}\right)+\frac{4}{m_{g}^{2}} \bar{\partial}\left(\Omega_{g} \partial \Omega_{g}^{-1}\right)=v^{-2} H_{0} H_{0}^{\dagger} \Omega_{g}^{-1} \Omega_{e}^{-1}-\mathbf{1}_{N}
$$

where $\Omega_{e}\left(\propto \mathbf{1}_{N}\right)$ and $\Omega_{g} \in S L(N, \mathbb{C})$ are positive-definite Hermitian matrices defined by

$$
\Omega_{e} \equiv S_{e} S_{e}^{\dagger}, \quad \Omega_{g} \equiv S_{g} S_{g}^{\dagger} .
$$

We call Eq. (2.13) the "master equation" for non-Abelian vortices.

By using the matrices $H_{0}(z), \Omega_{e}$ and $\Omega_{g}$, the BPS equations can be solved by the following procedure. Taking an arbitrary holomorphic matrix $H_{0}(z)$, we solve the master equation (2.13) in terms of $\Omega_{e}$ and $\Omega_{g}$ with the boundary conditions such that the vacuum equation (2.5) is satisfied at spatial infinity $|z| \rightarrow \infty$. Explicitly, they are given by

$$
\Omega_{e} \rightarrow v^{-2}\left|\operatorname{det} H_{0}\right|^{\frac{2}{N}} \mathbf{1}_{N}, \quad \Omega_{g} \rightarrow\left|\operatorname{det} H_{0}\right|^{-\frac{2}{N}} H_{0} H_{0}^{\dagger} .
$$

From the positive-definite hermitian matrices $\Omega_{e}=S_{e} S_{e}^{\dagger}$ and $\Omega_{g}=S_{g} S_{g}^{\dagger}$, the matrices $S_{e}$ and $S_{g}$ can be determined uniquely up to $U(1) \times S U(N)_{C}$ gauge transformation $S_{e} \rightarrow S_{e} e^{-i \alpha}, S_{g} \rightarrow$ $S_{g} U^{-1}$. Then, the physical fields can be obtained via the relations Eq. (2.10), Eq. (2.11) and Eq. (2.12).

As a consequence of the definitions (2.10), (2.11) and (2.12), the master equation (2.13) possesses a symmetry under the so-called " $V$-transformation" [21, 24]

$$
S_{g} S_{e} \rightarrow V(z) S_{g} S_{e}, \quad H_{0}(z) \rightarrow V(z) H_{0}(z),
$$


where $V(z) \in G L(N, \mathbb{C})$ is an arbitrary non-singular matrix holomorphic in $z$. Since the physical fields $w_{\mu}, W_{\mu}$ and $H$ are invariant under $V$-transformations, (2.16) defines an equivalence relation on the set of holomorphic matrices $H_{0}(z)$

$$
H_{0}(z) \sim V(z) H_{0}(z)
$$

There exists a one-to-one correspondence between the equivalence classes $H_{0} \sim V H_{0}$ and points on the moduli space of the BPS vortices [24, 21, 19, 27]. In this sense, we call $H_{0}(z)$ the "moduli matrix" and the parameters contained in $H_{0}(z)$ are identified with the moduli parameters of the BPS configurations. For example, the vortex positions for a given moduli matrix $H_{0}(z)$ can be determined as follows. Since a part of gauge symmetry is restored inside the vortex core, the vortex positions can be defined as those points on the complex plane at which the rank of the matrix $H$ becomes smaller than $N$, namely they can be determined as the zeros of the holomorphic polynomial $\operatorname{det} H_{0}$.

\subsection{Single vortex configurations}

As an example, let us consider configurations of a single vortex located at $z=z_{0}$. Since zeros of the polynomial det $H_{0}$ correspond to the vortex position, we consider the set of moduli matrices whose determinant is $\operatorname{det} H_{0}=z-z_{0}$. For example, in the case of $N=2$, any moduli matrix with det $H_{0}=z-z_{0}$ is $V$-equivalent to the moduli matrix of the form [22]

$$
H_{0}=\left(\begin{array}{cc}
z-z_{0} & 0 \\
-\beta & 1
\end{array}\right) \sim\left(\begin{array}{cc}
1 & -\tilde{\beta} \\
0 & z-z_{0}
\end{array}\right), \quad \tilde{\beta}=\frac{1}{\beta} .
$$

In addition to the translational moduli parameter $z_{0}$, there exists one parameter $\beta$ that can be viewed as an inhomogeneous coordinate of $\mathbb{C} P^{1}$. This internal degree of freedom, which is called the orientation, corresponds to the Nambu-Goldstone zero mode of $S U(2)_{C+F}$ symmetry broken by the vortex. The homogeneous coordinate of $\mathbb{C} P^{1}$ can also be extracted from the moduli matrix as follows. Since rank $H_{0}$ drops at $z=z_{0}$, there exists an eigenvector of $H_{0}$ with the null eigenvalue at $z=z_{0}$. In other words, there exists a constant $N$-vector $\phi$ such that

$$
\left.\left(H_{0} \phi\right)\right|_{z=z_{0}}=0, \quad \phi \propto\left(\begin{array}{c}
1 \\
\beta
\end{array}\right) .
$$

The vector $\phi$ is called the orientational vector and corresponds to the homogeneous coordinates of $\mathbb{C} P^{1}$. In the case of general $N$, a single vortex configuration breaks $S U(N)_{C+F}$ down to $S U(N-1) \times U(1)$, so that the orientational moduli space is

$$
\mathbb{C} P^{N-1}=\frac{S U(N)}{S U(N-1) \times U(1)} .
$$

In this case, the generic moduli matrix with $\operatorname{det} H_{0}=z-z_{0}$ is equivalent to

$$
H_{0}=\left(\begin{array}{cc}
z-z_{0} & 0 \\
-\vec{\beta} & \mathbf{1}_{N-1}
\end{array}\right)
$$


where $N-1$ parameters $\vec{\beta}$ are the inhomogeneous coordinates parameterizing the internal orientation $\mathbb{C} P^{N-1}$. As in the case of $N=2$, the orientational vector can also be defined by $\left.\left(H_{0} \phi\right)\right|_{z=z_{0}}=0$, corresponding to the homogeneous coordinates of $\mathbb{C} P^{N-1}$.

Next, let us briefly review some properties of the solution of the master equation (2.13) for the single vortex configuration [30]. First, note that the moduli matrix Eq. (2.21) can be rewritten as

$$
H_{0}=V(z)\left(\begin{array}{cc}
z-z_{0} & 0 \\
0 & \mathbf{1}_{N-1}
\end{array}\right) U
$$

where the matrices $V(z) \in S L(N, \mathbb{C})$ and $U \in S U(N)_{F}$ are given by

$$
\begin{aligned}
& V=\left(\begin{array}{cc}
\frac{1}{\sqrt{1+|\beta|^{2}}} & -\frac{\left(z-z_{0}\right) \vec{\beta}^{\dagger}}{\sqrt{1+|\vec{\beta}|^{2}}} \\
0 & (\mathbf{1}-\mathbf{P})+\sqrt{1+|\beta|^{2}} \mathbf{P}
\end{array}\right), \\
& U=\left(\begin{array}{cc}
\frac{1}{\sqrt{1+|\beta|^{2}}} & \frac{\vec{\beta}^{\dagger}}{\sqrt{1+|\beta|^{2}}} \\
-\frac{\vec{\beta}}{\sqrt{1+|\beta|^{2}}} & (\mathbf{1}-\mathbf{P})+\frac{1}{\sqrt{1+|\beta|^{2}}} \mathbf{P}
\end{array}\right) .
\end{aligned}
$$

and the $(N-1)$-by- $(N-1)$ matrix $\mathbf{P}$ is defined by

$$
\mathbf{P} \equiv \frac{\vec{\beta} \vec{\beta}^{\dagger}}{|\vec{\beta}|^{2}}
$$

It is convenient to use the following ansatz for $\Omega_{e}$ and $\Omega_{g}$

$$
\Omega_{e}=v^{-2} e^{\psi_{e} \mathbf{1}_{N}}, \quad \Omega_{g}=V e^{\psi_{g} T} V^{\dagger}
$$

where $\psi_{e}$ and $\psi_{g}$ are smooth real functions and the matrix $T \in s u(N)$ is given by

$$
T \equiv \operatorname{diag}(N-1,-1, \cdots,-1) .
$$

Then, the master equation (2.13) reduces to the following two equations for the functions $\psi_{e}$ and $\psi_{g}$

$$
\begin{aligned}
& \frac{4 N}{m_{e}^{2}} \partial \bar{\partial} \psi_{e}=e^{\psi_{g}-\psi_{e}}\left(1-\left|z-z_{0}\right|^{2} e^{-N \psi_{g}}\right)+N\left(1-e^{\psi_{g}-\psi_{e}}\right) \\
& \frac{4 N}{m_{g}^{2}} \partial \bar{\partial} \psi_{g}=e^{\psi_{g}-\psi_{e}}\left(1-\left|z-z_{0}\right|^{2} e^{-N \psi_{g}}\right) .
\end{aligned}
$$

The boundary conditions for $\psi_{e}$ and $\psi_{g}$ can be read from Eq. (2.15) as

$$
\psi_{e} \rightarrow \frac{1}{N} \log \left|z-z_{0}\right|^{2}, \quad \psi_{g} \rightarrow \frac{1}{N} \log \left|z-z_{0}\right|^{2} .
$$

By using the relation Eq. (2.14) and choosing an appropriate gauge, we obtain the matrices $S_{e}$ and $S_{g}$ in terms of the functions $\psi_{e}$ and $\psi_{g}$ as

$$
S_{e}=v^{-1} e^{\frac{1}{2} \psi_{e} \mathbf{1}_{N}}, \quad S_{g}=V e^{\frac{1}{2} \psi_{g} T} U
$$


Then, the solution can be obtained through Eq. (2.11) and Eq. (2.12) as

$$
\begin{aligned}
H & =v e^{-\frac{1}{2}\left(\psi_{e} \mathbf{1}_{N}+\psi_{g} T_{\beta}\right)} e^{\frac{1}{N}\left(\mathbf{1}_{N}+T_{\beta}\right) \log \left(z-z_{0}\right)}, \\
w_{\bar{z}} t^{0} & =-\frac{i}{2} \bar{\partial} \psi_{e} 1_{N}, \\
W_{\bar{z}}^{a} t^{a} & =-\frac{i}{2} \bar{\partial} \psi_{g} T_{\beta},
\end{aligned}
$$

where we have defined the matrix $T_{\beta}$ by

$$
T_{\beta} \equiv U^{\dagger} T U=N \frac{\phi \phi^{\dagger}}{|\phi|^{2}}-\mathbf{1}_{N}, \quad \phi \propto\left(\begin{array}{c}
1 \\
\vec{\beta}
\end{array}\right) .
$$

Now let us look at the asymptotic forms of the single vortex solution. The functions $\psi_{e}$ and $\psi_{g}$ behave near the vortex core as

$$
\begin{aligned}
& \psi_{e}=a_{e}+\frac{m_{e}^{2}}{4 N}\left[e^{a_{g}-a_{e}}+N\left(1-e^{a_{g}-a_{e}}\right)\right]\left|z-z_{0}\right|^{2}+\mathcal{O}\left(\left|z-z_{0}\right|^{4}\right), \\
& \psi_{g}=a_{g}+\frac{m_{g}^{2}}{4 N} e^{a_{g}-a_{e}}\left|z-z_{0}\right|^{2}+\mathcal{O}\left(\left|z-z_{0}\right|^{4}\right),
\end{aligned}
$$

where $a_{e}$ and $a_{g}$ are constants. On the other hand, the asymptotic forms of the functions $11 \psi_{e}$ and $\psi_{g}$ for large $\left|z-z_{0}\right|$ are given by [30]

$$
\begin{aligned}
& \psi_{e}=\frac{1}{N} \log \left|z-z_{0}\right|^{2}+c_{e} K_{0}\left(m_{e}\left|z-z_{0}\right|\right)+\mathcal{O}\left(e^{-2 m_{e}\left|z-z_{0}\right|}, e^{-2 m_{g}\left|z-z_{0}\right|}\right), \\
& \psi_{g}=\frac{1}{N} \log \left|z-z_{0}\right|^{2}+c_{g} K_{0}\left(m_{g}\left|z-z_{0}\right|\right)+\mathcal{O}\left(e^{-2 m_{g}\left|z-z_{0}\right|}, e^{-\left(m_{e}+m_{g}\right)\left|z-z_{0}\right|}\right),
\end{aligned}
$$

where $K_{0}\left(m\left|z-z_{0}\right|\right)$ is the modified Bessel function of the second kind. The constants $c_{e}$ and $c_{g}$ depend on the ratio $m_{g} / m_{e}$ and $N$. In case of $m_{e} \geq 2 m_{g}$, the term proportional to $K_{0}\left(m_{e}\left|z-z_{0}\right|\right)$ is actually not dominant in Eq.(2.38) compared to the contribution of order $e^{-2 m_{g}\left|z-z_{0}\right|}$. We then concentrate on the case with $m_{e}<2 m_{g}$ so that the above form is the proper approximation. As we will see, however, Eq.(2.38) and $\mathrm{Eq(2.39)}$ ) are sufficient to determine the metric to the leading order even for the case $m_{e}>2 m_{g}$.

\section{Effective Lagrangian for non-Abelian local vortices}

In this section, we derive a formula for the metric on the moduli space of non-Abelian local vortices which generalizes the celebrated Samols' formula for Abelian vortices [1].

\subsection{Formula for the metric on the moduli space}

The moduli space of the BPS vortices is a Kähler manifold whose holomorphic coordinates are identified with the complex parameters contained in $H_{0}(z)$. The effective low-energy dynamics

\footnotetext{
1 The function $\psi_{g}, \psi_{e}$ of the $S U(N), U(1)$ parts here are related to those in Ref. [30] by $\psi_{g}^{\text {here }}=\frac{1}{N-1} \psi_{g}^{30}+$ $\frac{1}{N} \log \left|z-z_{0}\right|^{2}$, and $\psi_{e}^{\text {here }}=\psi_{e}^{[30]}+\frac{1}{N} \log \left|z-z_{0}\right|^{2}$. Consequently, the coefficient $c_{g}^{\text {here }}=c_{g}^{30} /(N-1)$.
} 
of the BPS vortices are described by an effective Lagrangian of the form

$$
L_{\mathrm{eff}}=g_{i j} \dot{\phi}^{i} \dot{\bar{\phi}}^{j}=\frac{\partial^{2} K}{\partial \phi^{i} \partial \bar{\phi}^{j}} \dot{\phi}^{i} \dot{\bar{\phi}}^{j}
$$

where $\phi^{i}$ are the holomorphic coordinates, $g_{i \bar{j}}$ is the metric of the moduli space of non-Abelian vortices, and $K$ is the Kähler potential. By using the moduli matrix $H_{0}(z)$ and the solution $\left(\Omega_{e}, \Omega_{g}\right)$ of the master equation (2.13), the Kähler potential of the moduli space can be formally 2 written as 25$]$

$$
K=v^{2} \int d^{2} x \operatorname{Tr}\left[\frac{4}{m_{e}^{2}} \mathcal{K}\left(\Omega_{e}\right)+\frac{4}{m_{g}^{2}} \mathcal{K}\left(\Omega_{g}\right)+\log \left(\Omega_{e} \Omega_{g}\right)+v^{-2} H_{0} H_{0}^{\dagger} \Omega_{e}^{-1} \Omega_{g}^{-1}\right],
$$

where $\mathcal{K}(\Omega)$ is a quantity 3 which satisfies the following identity for a small variation $\Omega \rightarrow \Omega+\delta \Omega$

$$
\delta\left[\int d^{2} x \operatorname{Tr} \mathcal{K}(\Omega)\right]=\int d^{2} x \operatorname{Tr}\left[\bar{\partial}\left(\Omega \partial \Omega^{-1}\right) \delta \Omega \Omega^{-1}\right] .
$$

As a consequence, by varying the Kähler potential $K$ with respect to $\Omega_{e}$ and $\Omega_{g}$, we can show that $K$ is minimized by the solution of the master equation (2.13). Therefore, the derivatives of the Kähler potential with respect to the moduli parameters are given by

$$
\frac{\partial}{\partial \phi^{i}} K=\int d^{2} x \operatorname{Tr}\left(\frac{\partial}{\partial \phi^{i}} H_{0} H_{0}^{\dagger} \Omega_{e}^{-1} \Omega_{g}^{-1}\right)
$$

Note that $H_{0}^{\dagger}$ is anti-holomorphic in $\phi^{i}$. From this property of the Kähler potential, we obtain a simple form of the effective Lagrangian

$$
L_{\mathrm{eff}}=\frac{\partial^{2} K}{\partial \phi^{i} \partial \bar{\phi}^{j}} \dot{\phi}^{i} \dot{\bar{\phi}}^{j}=\int d^{2} x \delta_{t}^{\dagger} \operatorname{Tr}\left(\delta_{t} H_{0} H_{0}^{\dagger} \Omega_{g}^{-1} \Omega_{e}^{-1}\right)
$$

where the differential operators $\delta_{t}$ and $\delta_{t}^{\dagger}$ are defined by

$$
\delta_{t}=\dot{\phi}^{i} \frac{\partial}{\partial \phi^{i}}, \quad \delta_{t}^{\dagger}=\dot{\bar{\phi}}^{i} \frac{\partial}{\partial \bar{\phi}^{i}} .
$$

Now we rewrite the effective Lagrangian in terms of local data in the neighborhood of each vortex. Let us assume that $\operatorname{det} H_{0}$ has zeros at $z=z_{I}(I=1,2, \cdots)$, namely

$$
\operatorname{det} H_{0}(z)=\prod_{I=1}^{k}\left(z-z_{I}\right)
$$

2 To make the Kähler potential finite, we need to add counter terms which can be regarded as a Kähler transformation.

3 The explicit form of $\mathcal{K}(\Omega)$ is given by

$$
\operatorname{Tr} \mathcal{K}(\Omega)=\int_{0}^{1} d s \int_{0}^{s} d t \operatorname{Tr}\left[\bar{\partial} \omega e^{t \omega} \partial \omega e^{-t \omega}\right], \quad \omega \equiv \log \Omega
$$


Let $D_{\epsilon}^{I}$ be the disk of radius $\epsilon$ centered at $z=z_{I}$

$$
D_{\epsilon}^{I}=\left\{z \in \mathbb{C}|| z-z_{I} \mid<\epsilon\right\} .
$$

It is convenient to decompose the domain of integration $\mathbb{C}$ into the disks $D_{\epsilon}^{I}$ and their complement $\mathbb{C}-\bigcup D_{\epsilon}^{I}$. Since the integrand in (3.5) does not have any singularity, the integral over the disk $D_{\epsilon}^{I}$ vanishes in the zero-radius limit $\epsilon \rightarrow 0$

$$
\lim _{\epsilon \rightarrow 0} \int_{D_{\epsilon}^{I}} d^{2} x \delta_{t}^{\dagger} \operatorname{Tr}\left(\delta_{t} H_{0} H_{0}^{\dagger} \Omega_{g}^{-1} \Omega_{e}^{-1}\right)=0 .
$$

Therefore, the effective Lagrangian can be evaluated by integrating over $\mathbb{C}-\sum D_{\epsilon}^{I}$ and then taking $\epsilon \rightarrow 0$ limit

$$
L_{\mathrm{eff}}=\lim _{\epsilon \rightarrow 0} \int_{\mathbb{C}-\cup D_{\epsilon}^{I}} d^{2} x \delta_{t}^{\dagger} \operatorname{Tr}\left(\delta_{t} H_{0} H_{0}^{\dagger} \Omega_{g}^{-1} \Omega_{e}^{-1}\right) .
$$

Using the master equation (2.13) the integrand can be put in the form of a total derivative as

$$
\begin{aligned}
\delta_{t}^{\dagger} \operatorname{Tr}\left(\delta_{t} H_{0} H_{0}^{\dagger} \Omega_{g}^{-1} \Omega_{e}^{-1}\right) & =v^{2} \delta_{t}^{\dagger} \operatorname{Tr}\left[\delta_{t} H_{0} H_{0}^{-1}\left(\frac{4}{m_{g}^{2}} \bar{\partial}\left(\Omega_{g} \partial \Omega_{g}^{-1}\right)+\frac{4}{m_{e}^{2}} \bar{\partial}\left(\Omega_{e} \partial \Omega_{e}^{-1}\right)+\mathbf{1}_{N}\right)\right] \\
& =v^{2} \bar{\partial} \operatorname{Tr}\left[\delta_{t} H_{0} H_{0}^{-1} \delta_{t}^{\dagger}\left(\frac{4}{m_{g}^{2}} \Omega_{g} \partial \Omega_{g}^{-1}+\frac{4}{m_{e}^{2}} \Omega_{e} \partial \Omega_{e}^{-1}\right)\right],
\end{aligned}
$$

where we used the fact that $\delta_{t} H_{0} H_{0}^{-1}$ is holomorphic with respect to $z$ and the moduli parameters $\phi^{i}$ on $\mathbb{C}-\sum D_{\epsilon}^{I}$. For Stokes' theorem, the integral of Eq. (3.11) over $\mathbb{C}-\sum D_{\epsilon}^{I}$ can be replaced by an integral along the infinitely large circle $S_{\infty}^{1}$ and the boundaries of the disks $-\partial D_{\epsilon}^{I}$. Since the integrand falls off exponentially at spatial infinity, the contribution from $S_{\infty}^{1}$ vanishes and the effective Lagrangian becomes

$$
L_{\mathrm{eff}}=-\frac{v^{2}}{2 i} \sum_{I=1}^{k} \lim _{\epsilon \rightarrow 0} \int_{\partial D_{\epsilon}^{I}} d z \operatorname{Tr}\left[\delta_{t} H_{0} H_{0}^{-1} \delta_{t}^{\dagger}\left(\frac{4}{m_{g}^{2}} \Omega_{g} \partial \Omega_{g}^{-1}+\frac{4}{m_{e}^{2}} \Omega_{e} \partial \Omega_{e}^{-1}\right)\right] .
$$

Since each integral picks up the terms which behave as $1 /\left(z-z_{I}\right)$, it can be evaluated by expanding the integrand around $z=z_{I}$.

First let us consider the case where all the zeros of the polynomial det $H_{0}(z)$ are isolated. In this case, the matrix $\delta_{t} H_{0} H_{0}^{-1}$ has the first order pole at $z=z_{I}$

$$
\delta_{t} H_{0} H_{0}^{-1}=\frac{Q_{I}}{z-z_{I}}+\left\{\text { regular at } z=z_{I}\right\} .
$$

Since the remaining part of the integrand is non-singular, it can be expanded around $z=z_{I}$ as

$$
\delta_{t}^{\dagger}\left(\frac{4}{m_{g}^{2}} \Omega_{g} \partial \Omega_{g}^{-1}+\frac{4}{m_{e}^{2}} \Omega_{e} \partial \Omega_{e}^{-1}\right)=\dot{\bar{z}}_{I} C_{I}-\delta_{t}^{\dagger} B_{I}+\mathcal{O}\left(\left|z-z_{I}\right|\right),
$$

where the matrices $B_{I}$ and $C_{I}$ are defined by

$$
\begin{aligned}
B_{I} & \equiv-\lim _{z \rightarrow z_{I}}\left[\frac{4}{m_{g}^{2}} \Omega_{g} \partial \Omega_{g}^{-1}+\frac{4}{m_{e}^{2}} \Omega_{e} \partial \Omega_{e}^{-1}\right], \\
C_{I} & \equiv-\lim _{z \rightarrow z_{I}}\left[\frac{4}{m_{g}^{2}} \bar{\partial}\left(\Omega_{g} \partial \Omega_{g}^{-1}\right)+\frac{4}{m_{e}^{2}} \bar{\partial}\left(\Omega_{e} \partial \Omega_{e}^{-1}\right)\right] .
\end{aligned}
$$


Then, the effective Lagrangian can be written as

$$
L_{\mathrm{eff}}=-\pi v^{2} \sum_{I} \operatorname{Tr}\left[Q_{I}\left(\dot{\bar{z}}_{I} C_{I}-\delta_{t}^{\dagger} B_{I}\right)\right] .
$$

From the master equation (2.13), we evaluate $C_{I}$ :

$$
C_{I}=\mathbf{1}_{N}-\left.v^{-2} H_{0} H_{0}^{\dagger} \Omega_{g}^{-1} \Omega_{e}^{-1}\right|_{z=z_{I}} .
$$

Therefore, the effective Lagrangian reduces to

$$
L_{\mathrm{eff}}=\pi v^{2} \sum_{I}\left[\left|\dot{z}_{I}\right|^{2}+\operatorname{Tr}\left(Q_{I} \delta_{t}^{\dagger} B_{I}\right)\right],
$$

where we have used $\operatorname{Tr} Q_{I}=-\dot{z}_{I}$ and

$$
\left.Q_{I} H_{0} H_{0}^{\dagger} \Omega_{g}^{-1} \Omega_{e}^{-1}\right|_{z=z_{I}}=\lim _{z \rightarrow z_{I}}\left(z-z_{I}\right) \delta_{t} H_{0} H_{0}^{\dagger} \Omega_{g}^{-1} \Omega_{e}^{-1}=0 .
$$

Note the strong similarity between Eq. (3.19) and Samols' formula [1] (although the analogy between the definitions of our quantities $B_{I}$ and the corresponding ones of Samols is not complete, as it is made explicit in Section $4 \mathrm{Eq}$. (4.13)).

\subsection{Example: single vortex}

As an example, let us consider the single vortex configuration. For the moduli matrix Eq. (2.21), the matrix $Q$ can be calculated as

$$
Q=\lim _{z \rightarrow z_{0}}\left(z-z_{0}\right) \delta_{t} H_{0} H_{0}^{-1}=\left(\begin{array}{cc}
-\dot{z}_{0} & 0 \\
-\dot{\vec{\beta}} & \mathbf{0}_{N-1}
\end{array}\right) .
$$

On the other hand, the matrix $B$ can be calculated by using the ansatz Eq. (2.26) as

$$
B=-\lim _{z \rightarrow z_{0}}\left[\frac{4}{m_{g}^{2}} \Omega_{g} \partial \Omega_{g}^{-1}+\frac{4}{m_{e}^{2}} \Omega_{e} \partial \Omega_{e}^{-1}\right]=-\frac{4}{m_{g}^{2}} \lim _{z \rightarrow z_{0}} V \partial V^{-1} .
$$

Note that both functions $\psi_{e}$ and $\psi_{g}$ satisfy $\lim _{z \rightarrow z_{0}} \partial \psi_{e}=\lim _{z \rightarrow z_{0}} \partial \psi_{g}=0$ (see Eq. (2.36) and Eq. (2.37) ). From the explicit form of the matrix $V$ given in Eq. (2.23), we find that the matrix $B$ is given by

$$
B=\frac{4}{m_{g}^{2}}\left(\begin{array}{cc}
0 & -\frac{\vec{\beta}^{\dagger}}{1+|\vec{\beta}|^{2}} \\
0 & \mathbf{0}_{N-1}
\end{array}\right) .
$$

Substituting $Q$ and $B$ into (3.19), we obtain the following effective Lagrangian for a single nonAbelian vortex

$$
L_{\mathrm{eff}}=\pi v^{2}\left|\dot{z}_{0}\right|^{2}+\frac{4 \pi}{g^{2}} \dot{\vec{\beta}}^{\dagger} \cdot \frac{\left(1+|\vec{\beta}|^{2}\right) \mathbf{1}-\vec{\beta} \vec{\beta}^{\dagger}}{\left(1+|\vec{\beta}|^{2}\right)^{2}} \cdot \dot{\vec{\beta}}
$$




\subsection{Coincident case}

Next, let us consider the case of coincident vortices. If det $H_{0}(z)$ has $k_{I}$-th order zero at $z=z_{I}$, the matrix $\delta_{t} H_{0} H_{0}^{-1}$ has the following Laurent series expansion

$$
\delta_{t} H_{0} H_{0}^{-1}=\sum_{p=1}^{k_{I}} \frac{Q_{p}^{I}}{\left(z-z_{I}\right)^{p}}+\left\{\text { regular at } z=z_{I}\right\} .
$$

On the other hand, the remaining part of the integrand is non-singular and can be expanded as

$$
\delta_{t}^{\dagger}\left(\frac{4}{m_{g}^{2}} \Omega_{g} \partial \Omega_{g}^{-1}+\frac{4}{m_{e}^{2}} \Omega_{e} \partial \Omega_{e}^{-1}\right)=\sum_{p, q=0}^{\infty} R_{p, q}^{I}\left(z-z_{I}\right)^{p}\left(\bar{z}-\bar{z}_{I}\right)^{q} .
$$

Then, the effective Lagrangian can be written in terms of the coefficients $Q_{p}$ and $R_{p, q}^{I}$ as

$$
L_{\mathrm{eff}}=-\pi v^{2} \sum_{I} \sum_{p=1}^{k_{I}} \operatorname{Tr}\left(Q_{p}^{I} R_{p-1,0}^{I}\right)
$$

\section{Asymptotic metric for well-separated vortices}

In this section, we consider the asymptotic form of the metric on the moduli space for $k$ wellseparated non-Abelian vortices by generalizing the results for Abelian vortices [13].

\subsection{Asymptotic metric for Abelian vortices}

First, let us rederive the effective Lagrangian for well-separated vortices in the $N=1$ (Abelian) theory [13]. Our approach here has essentially the same spirit of Section 2 in [13], however our use of complex notation will make more transparent some properties retained by the solutions of the linearized vortex equation, which are crucial for the derivation of the result.

In this case, the moduli matrix $H_{0}(z)$ is a holomorphic polynomial of $z$ and can be written as

$$
H_{0}(z)=\prod_{I=1}^{k}\left(z-z_{I}\right)
$$

where $k$ is the number of vortices and $z=z_{I}(I=1, \cdots, k)$ are the positions of vortices. To calculate the asymptotic metric for well-separated vortices, it is convenient to define the function $\hat{\psi}$ by

$$
\hat{\psi} \equiv \log \Omega_{e}-\log \left|H_{0}\right|^{2}+\log v^{2}
$$

Then, the master equation (2.13) can be rewritten in terms of $\hat{\psi}$ as

$$
\frac{4}{m_{e}^{2}} \partial \bar{\partial} \hat{\psi}+e^{-\hat{\psi}}-1=-\frac{4 \pi}{m_{e}^{2}} \sum_{I=1}^{k} \delta^{2}\left(z-z_{I}\right)
$$


where boundary condition for large $|z|$ is given by $\hat{\psi} \rightarrow 0$. Let us consider the linearized equation for the small fluctuation $\Delta \hat{\psi}$ around the background solution $\hat{\psi}$

$$
\left(\frac{4}{m_{e}^{2}} \partial \bar{\partial}-e^{-\hat{\psi}}\right) \Delta \hat{\psi}=0, \quad z \neq z_{I}
$$

Let $\Delta_{1} \hat{\psi}$ and $\Delta_{2} \hat{\psi}$ be linearly independent solutions of the linearized equation. Then, the "current" $\left(j_{z}, j_{\bar{z}}\right)$ defined by

$$
j_{z} \equiv \frac{4}{m_{e}^{2}}\left(\Delta_{1} \hat{\psi}\right) \partial\left(\Delta_{2} \hat{\psi}\right)-(1 \leftrightarrow 2), \quad j_{\bar{z}} \equiv \frac{4}{m_{e}^{2}}\left(\Delta_{1} \hat{\psi}\right) \bar{\partial}\left(\Delta_{2} \hat{\psi}\right)-(1 \leftrightarrow 2),
$$

satisfies the following "conservation law" except at the vortex positions

$$
\bar{\partial} j_{z}+\partial j_{\bar{z}}=0, \quad z \neq z_{I}
$$

Therefore, the contour integrals

$$
q_{I} \equiv \frac{1}{2 \pi i} \oint_{C_{I}}\left(d z j_{z}-d \bar{z} j_{\bar{z}}\right), \quad(I=1, \cdots, k)
$$

are invariant under continuous deformations of the contour $C_{I}$ surrounding $z=z_{I}$. This property of the invariants $q_{I}$ can be used to relate the local data in the neighborhood of each vortex to the asymptotic data.

As the first solution of the linearized equation, let us take the derivative of $\hat{\psi}$ with respect to the $I$-th moduli parameter $z_{I}$ (no sum over $I$ is implied)

$$
\Delta_{1} \hat{\psi}=\delta_{t} \hat{\psi} \equiv \dot{z}_{I} \frac{\partial \hat{\psi}}{\partial z_{I}}
$$

Note that $\delta_{t} \hat{\psi}$ satisfies the linearized equation (4.4) except at $z=z_{I}$. As the second solution, we take the difference of the full $k$-vortex solution $\hat{\psi}$ and the single vortex solution $\hat{\psi}_{I}$ satisfying

$$
\frac{4}{m_{e}^{2}} \partial \bar{\partial} \hat{\psi}_{I}+e^{-\hat{\psi}_{I}}+1=-\frac{4}{m_{e}^{2}} \delta^{2}\left(z-z_{I}\right) .
$$

Note that the difference of the solutions

$$
\Delta_{2} \hat{\psi}=\hat{\psi}-\hat{\psi}_{I}
$$

is an approximate solution of the linearized equation (4.4) since both $\hat{\psi}$ and $\hat{\psi}_{I}$ satisfy the same equation except at $z=z_{J}(J \neq I)$ and furthermore their difference is small if $\left|z-z_{J}\right| \gg m_{e}^{-1}$ for all $J \neq I$.

Let us first calculate the contour integral by taking the zero radius limit of the circular contour $C_{I}$ surrounding $z=z_{I}$. Since the integral picks up the terms which behave like $1 /\left(z-z_{I}\right)$ and $1 /\left(\bar{z}-\bar{z}_{I}\right)$ in the zero radius limit, it can be evaluated by expanding the integrand around $z=z_{I}$

$$
\begin{aligned}
\hat{\psi} & =-\log \left|z-z_{I}\right|^{2}+a_{I}+b_{I}\left(z-z_{I}\right)+\bar{b}_{I}\left(\bar{z}-\bar{z}_{I}\right)+\frac{m_{e}^{2}}{4}\left|z-z_{I}\right|^{2}+\cdots \\
\hat{\psi}_{I} & =-\log \left|z-z_{I}\right|^{2}+\tilde{a}_{I}+\frac{m_{e}^{2}}{4}\left|z-z_{I}\right|^{2}+\cdots
\end{aligned}
$$

\footnotetext{
${ }^{4}$ Note that $j_{z}$ and $j_{\bar{z}}$ are not complex conjugate in general.
} 
where the coefficients $b_{I}$ are related to $B_{I}=\left.\frac{4}{m_{e}^{2}} \partial \log \Omega_{e}\right|_{z=z_{I}}$ as 5

$$
b_{I} \equiv \frac{m_{e}^{2}}{4} B_{I}-\sum_{J \neq I} \frac{1}{z_{I}-z_{J}} \text {. }
$$

Therefore, $\Delta_{1} \hat{\psi}$ and $\Delta_{2} \hat{\psi}$ behave around $z=z_{I}$ as

$$
\begin{aligned}
\Delta_{1} \hat{\psi} & =\frac{\dot{z}_{I}}{z-z_{I}}+\frac{\partial a_{I}}{\partial z_{I}} \dot{z}_{I}-b_{I} \dot{z}_{I}-\frac{m_{e}^{2}}{4} \dot{z}_{I}\left(\bar{z}-\bar{z}_{I}\right)+\cdots \\
\Delta_{2} \hat{\psi} & =a_{I}-\tilde{a}_{I}+b_{I}\left(z-z_{I}\right)+\bar{b}_{I}\left(\bar{z}-\bar{z}_{I}\right)+\cdots
\end{aligned}
$$

From this behavior of the functions $\Delta_{1} \hat{\psi}$ and $\Delta_{2} \hat{\psi}$, we obtain

$$
q_{I}=\frac{4}{m_{e}^{2}} 2 \dot{z}_{I} b_{I}=2 \dot{z}_{I}\left(B_{I}-\frac{4}{m_{e}^{2}} \sum_{J \neq I} \frac{1}{z_{I}-z_{J}}\right) .
$$

On the other hand, the integral can also be evaluated along a large contour on which the solutions can be approximated by their asymptotic forms

$$
\begin{aligned}
\hat{\psi} & =\sum_{J=1}^{k} c_{e} K_{0}\left(m_{e}\left|z-z_{J}\right|\right)+\cdots, \\
\hat{\psi}_{I} & =c_{e} K_{0}\left(m_{e}\left|z-z_{I}\right|\right)+\cdots,
\end{aligned}
$$

Therefore, $\Delta_{1} \hat{\psi}$ and $\Delta_{2} \hat{\psi}$ have the following asymptotic forms

$$
\begin{aligned}
\Delta_{1} \hat{\psi} & =c_{e} \dot{z}_{I} \frac{\partial}{\partial z_{I}} K_{0}\left(m_{e}\left|z-z_{I}\right|\right)+\cdots, \\
\Delta_{2} \hat{\psi} & =\sum_{J \neq I} c_{e} K_{0}\left(m_{e}\left|z-z_{J}\right|\right)+\cdots
\end{aligned}
$$

From these asymptotic forms of the functions $\Delta_{1} \hat{\psi}$ and $\Delta_{2} \hat{\psi}$, we obtain

$$
\begin{aligned}
q_{I} & =\frac{4 c_{e}^{2}}{m_{e}^{2}} \dot{z}_{I} \frac{\partial}{\partial z_{I}} \sum_{J \neq I} \int\left[\left(\frac{d z}{2 \pi i} K_{0}\left(m_{e}\left|z-z_{I}\right|\right) \partial K_{0}\left(m_{e}\left|z-z_{J}\right|\right)-(I \leftrightarrow J)\right)+(\text { c.c. })\right] \\
& =\frac{4 c_{e}^{2}}{m_{e}^{2}} \dot{z}_{I} \frac{\partial}{\partial z_{I}} \sum_{J \neq I} K_{0}\left(m_{e}\left|z_{I}-z_{J}\right|\right) .
\end{aligned}
$$

Comparing two expressions of $q_{I}$, we can relate the local data and the asymptotic data as

$$
\dot{z}_{I} B_{I}=\sum_{J \neq I}\left(\frac{2 c_{e}^{2}}{m_{e}^{2}} \dot{z}_{I} \frac{\partial}{\partial z_{I}} K_{0}\left(m_{e}\left|z_{I}-z_{J}\right|\right)+\frac{4}{m_{e}^{2}} \frac{\dot{z}_{I}}{z_{I}-z_{J}}\right) .
$$

Since the constant $Q_{I}$ are given by

$$
Q_{I}=\lim _{z \rightarrow z_{I}}\left(z-z_{I}\right) \delta_{t} H_{0} H_{0}^{-1}=-\dot{z}_{I}
$$

\footnotetext{
${ }^{5}$ Here the difference between our $B_{I}$ and Samols' $b_{I}$ is evident, however it is obvious that if one operates with $\delta_{t}^{\dagger}$, like in the final formula for the metric, the outcome is the same.
} 
we obtain the effective Lagrangian from Eq. (3.19) as

$$
L_{\mathrm{eff}}=\pi v^{2}\left[\sum_{I=1}^{k}\left|\dot{z}_{I}\right|^{2}-\frac{c_{e}^{2}}{4} \sum_{I \neq J} K_{0}\left(m_{e}\left|z_{I}-z_{J}\right|\right)\left|\dot{z}_{I}-\dot{z}_{J}\right|^{2}\right]
$$

where we have used (no sum over $I$ is implied)

$$
4 \frac{\partial}{\partial z_{I}} \frac{\partial}{\partial \bar{z}_{I}} K_{0}\left(m_{e}\left|z_{I}-z_{J}\right|\right)=-4 \frac{\partial}{\partial z_{I}} \frac{\partial}{\partial \bar{z}_{J}} K_{0}\left(m_{e}\left|z_{I}-z_{J}\right|\right)=m_{e}^{2} K_{0}\left(m_{e}\left|z_{I}-z_{J}\right|\right) .
$$

\subsection{Asymptotic metric for non-Abelian vortices}

The most generic form moduli matrix for multi-vortex configuration is given by

$$
H_{0}=\left(\begin{array}{cc}
P(z) & 0 \\
\vec{R}(z) & \mathbf{1}
\end{array}\right), \quad P(z)=\prod_{I=1}^{k}\left(z-z_{I}\right), \quad \vec{R}(z)=-\sum_{I=1}^{k} \vec{\beta}_{I} \prod_{J \neq I} \frac{z-z_{J}}{z_{I}-z_{J}} .
$$

There are $N$ complex moduli parameters for each vortex. One is the position moduli $z_{I}$ and the others are the orientational moduli $\vec{\beta}_{I}$. Note that the orientational vector for the vortex located at $z=z_{I}$ is

$$
\phi \propto\left(\begin{array}{c}
1 \\
\vec{\beta}_{I}
\end{array}\right), \quad\left(\left.\because H_{0} \phi\right|_{z=z_{I}}=0\right) .
$$

To calculate the asymptotic metric for well-separated vortices, it is convenient to redefine the matrices $\Omega_{g}$ and $\Omega_{e}$ by

$$
\begin{aligned}
& \hat{\Omega}_{g} \equiv\left|\operatorname{det} H_{0}\right|^{\frac{2}{N}} H_{0}^{-1} \Omega_{g} H_{0}^{\dagger-1} \\
& \hat{\Omega}_{e} \equiv v^{2}\left|\operatorname{det} H_{0}\right|^{-\frac{2}{N}} \Omega_{e} .
\end{aligned}
$$

The matrices $\hat{\Omega}_{e}$ and $\hat{\Omega}_{g}$ satisfy

$$
\frac{4}{m_{e}^{2}} \bar{\partial}\left(\hat{\Omega}_{e} \partial \hat{\Omega}_{e}^{-1}\right)+\frac{4}{m_{g}^{2}} \bar{\partial}\left(\hat{\Omega}_{g} \partial \hat{\Omega}_{g}^{-1}\right)-\hat{\Omega}_{g}^{-1} \hat{\Omega}_{e}^{-1}+\mathbf{1}_{N}=0, \quad z \neq z_{I}
$$

The boundary conditions Eq. (2.15) can be translated into those for $\hat{\Omega}_{e}$ and $\hat{\Omega}_{g}$ as

$$
\hat{\Omega}_{e} \rightarrow \mathbf{1}_{N}, \quad \hat{\Omega}_{g} \rightarrow \mathbf{1}_{N} .
$$

For a given background solution $\left(\hat{\Omega}_{e}, \hat{\Omega}_{g}\right)$, let us consider the following linearized equation for small fluctuations $\left(\Delta \hat{\Omega}_{e}, \Delta \hat{\Omega}_{g}\right)$

$$
\frac{4}{m_{e}^{2}} \bar{\partial}\left[\Delta\left(\hat{\Omega}_{e} \partial \hat{\Omega}_{e}^{-1}\right)\right]+\frac{4}{m_{g}^{2}} \bar{\partial}\left[\Delta\left(\hat{\Omega}_{g} \partial \hat{\Omega}_{g}^{-1}\right)\right]-\hat{\Omega}_{g}^{-1} \hat{\Omega}_{e}^{-1}\left(\hat{\Omega}_{g} \Delta \hat{\Omega}_{g}^{-1}+\hat{\Omega}_{e} \Delta \hat{\Omega}_{e}^{-1}\right)=0, \quad z \neq z_{I}
$$

As in the case of the Abelian vortices, we can define the "conserved current" $\left(j_{z}, j_{\bar{z}}\right)$ from the solutions of the linearized equation (4.32) as

$$
\begin{aligned}
& j_{z}=\operatorname{Tr}\left[\frac{4}{m_{e}^{2}}\left(\hat{\Omega}_{e} \Delta_{1} \hat{\Omega}_{e}^{-1}\right) \Delta_{2}\left(\hat{\Omega}_{e} \partial \hat{\Omega}_{e}^{-1}\right)+\frac{4}{m_{g}^{2}}\left(\hat{\Omega}_{g} \Delta_{1} \hat{\Omega}_{g}^{-1}\right) \Delta_{2}\left(\hat{\Omega}_{g} \partial \hat{\Omega}_{g}^{-1}\right)\right]-(1 \leftrightarrow 2), \\
& j_{\bar{z}}=\operatorname{Tr}\left[\frac{4}{m_{e}^{2}}\left(\Delta_{1} \hat{\Omega}_{e}^{-1} \hat{\Omega}_{e}\right) \Delta_{2}\left(\bar{\partial} \hat{\Omega}_{e}^{-1} \hat{\Omega}_{e}\right)+\frac{4}{m_{g}^{2}}\left(\Delta_{1} \hat{\Omega}_{g}^{-1} \hat{\Omega}_{g}\right) \Delta_{2}\left(\bar{\partial} \hat{\Omega}_{g}^{-1} \hat{\Omega}_{g}\right)\right]-(1 \leftrightarrow 2) .
\end{aligned}
$$


As the solution of the linearized equation, we take the derivatives of $\hat{\Omega}_{e}$ and $\hat{\Omega}_{g}$ with respect to the moduli parameters

$$
\Delta_{1} \hat{\Omega}_{e}=\delta_{t} \hat{\Omega}_{e}, \quad \Delta_{1} \hat{\Omega}_{g}=\delta_{t} \hat{\Omega}_{g}
$$

and the difference of the full solution $\left(\hat{\Omega}_{e}, \hat{\Omega}_{g}\right)$ and the single vortex solution $\left(\hat{\Omega}_{e I}, \hat{\Omega}_{g I}\right)$

$$
\Delta_{2} \hat{\Omega}_{e}=\hat{\Omega}_{e}-\hat{\Omega}_{e I}, \quad \Delta_{2} \hat{\Omega}_{g}=\hat{\Omega}_{g}-\hat{\Omega}_{g I}
$$

From the fact that the current $\left(j_{z}, j_{\bar{z}}\right)$ satisfies the conservation law $\bar{\partial} j_{z}+\partial j_{\bar{z}}=0$, the contour integrals

$$
q_{I}=\frac{1}{2 \pi i} \oint_{C_{I}}\left(d z j_{z}-d \bar{z} j_{\bar{z}}\right), \quad(I=1,2, \cdots, k)
$$

are invariant under the continuous deformation of the contour $C_{I}$ surrounding $z=z_{I}$. By expanding the integrand around $z=z_{I}$ and picking up the terms which behave like $1 /\left(z-z_{I}\right)$ and $1 /\left(\bar{z}-\bar{z}_{I}\right)$, the contour integral $q_{I}$ can be evaluated in the zero-radius limit as

$$
\begin{aligned}
q_{I} & =2 \operatorname{Tr}\left[Q_{I} B_{I}\right]-2 \operatorname{Tr}\left[Q_{I}^{\text {single }} B_{I}^{\text {single }}\right]+f\left(z_{J}, \beta_{J}\right) \\
& =2 \operatorname{Tr}\left[Q_{I} B_{I}\right]-\frac{8}{m_{g}^{2}} \frac{\vec{\beta}_{I}^{\dagger} \cdot \dot{\vec{\beta}}_{I}}{1+\left|\vec{\beta}_{I}\right|^{2}}+f\left(z_{J}, \beta_{J}\right),
\end{aligned}
$$

where $f\left(z_{J}, \beta_{J}\right)$ is a holomorphic function of the moduli parameters. Next, let us evaluate the integral along a large contour on which the solutions can be approximated by their asymptotic forms Eq.(2.38) and Eq.(2.39)

$$
\begin{aligned}
& \hat{\Omega}_{e}=\mathbf{1}_{N}+c_{e} \sum_{I=1}^{k} K_{0}\left(m_{e}\left|z-z_{I}\right|\right) \mathbf{1}_{N}+\cdots, \\
& \hat{\Omega}_{g}=\mathbf{1}_{N}+c_{g} \sum_{I=1}^{k} K_{0}\left(m_{g}\left|z-z_{I}\right|\right) T_{\beta_{I}}+\cdots .
\end{aligned}
$$

By using these asymptotic forms and the formula

$$
K_{0}\left(m\left|z_{I}-z_{J}\right|\right)=\int_{C_{I}} \frac{d z}{2 \pi i}\left[K_{0}\left(m\left|z-z_{I}\right|\right) \partial K_{0}\left(m\left|z-z_{J}\right|\right)-(I \leftrightarrow J)\right]+(\text { c.c. }),
$$

we obtain

$$
q_{I}=\delta_{t}\left[\frac{4 N c_{e}^{2}}{m_{e}^{2}} \sum_{J \neq I} K_{0}\left(m_{e}\left|z_{I}-z_{J}\right|\right)+\frac{4 N c_{g}^{2}}{m_{g}^{2}} \sum_{J \neq I} \Theta_{I J} K_{0}\left(m_{g}\left|z_{I}-z_{J}\right|\right)\right]
$$

where $\Theta_{I J}$ is defined by

$$
\Theta_{I J} \equiv \frac{1}{N} \operatorname{Tr}\left(T_{\beta_{I}} T_{\beta_{J}}\right)=N \frac{\left|1+\vec{\beta}_{I} \cdot \vec{\beta}_{J}^{\dagger}\right|^{2}}{\left(1+\left|\vec{\beta}_{I}\right|^{2}\right)\left(1+\left|\vec{\beta}_{J}\right|^{2}\right)}-1
$$


Comparing Eq. (4.38) and Eq. (4.42), we find that

$$
\begin{aligned}
\operatorname{Tr}\left[Q_{I} \delta_{t}^{\dagger} B_{I}\right]= & -\delta_{t}^{\dagger} \delta_{t}\left[\frac{2 N c_{e}^{2}}{m_{e}^{2}} \sum_{J \neq I} K_{0}\left(m_{e}\left|z_{I}-z_{J}\right|\right)+\frac{2 N c_{g}^{2}}{m_{g}^{2}} \sum_{J \neq I} \Theta_{I J} K_{0}\left(m_{g}\left|z_{I}-z_{J}\right|\right)\right] \\
& +\delta_{t}^{\dagger} \delta_{t}\left[\frac{4}{m_{g}^{2}} \log \left(1+\left|\vec{\beta}_{I}\right|^{2}\right)\right]
\end{aligned}
$$

Then, we obtain the asymptotic effective Lagrangian form Eq. (3.19)

$$
L_{\mathrm{eff}}=\pi v^{2}\left(\sum_{I=1}^{k}|\dot{z}|^{2}+\operatorname{Tr}\left[Q_{I} \delta_{t}^{\dagger} B_{I}\right]\right)=\delta_{t}^{\dagger} \delta_{t} K
$$

where the Kähler potential is given by

$$
\begin{aligned}
K & =\sum_{I=1}^{k}\left[\pi v^{2}\left|z_{I}\right|^{2}+\frac{4 \pi}{g^{2}} \log \left(1+\left|\vec{\beta}_{I}\right|^{2}\right)\right]-\sum_{I \neq J}\left[\frac{2 \pi v^{2} N c_{e}^{2}}{m_{e}^{2}} K_{0}\left(m_{e}\left|z_{I}-z_{J}\right|\right)\right] \\
& -\sum_{I \neq J}\left[\frac{2 \pi v^{2} N c_{g}^{2}}{m_{g}^{2}}\left(N \frac{\left|1+\vec{\beta}_{I} \cdot \vec{\beta}_{J}^{\dagger}\right|^{2}}{\left(1+\left|\vec{\beta}_{I}\right|^{2}\right)\left(1+\left|\vec{\beta}_{J}\right|^{2}\right)}-1\right) K_{0}\left(m_{g}\left|z_{I}-z_{J}\right|\right)\right] .
\end{aligned}
$$

The last two terms describe leading interactions between different vortices. In homogeneous coordinates ( $N$-vector) $\vec{\phi}_{I}$ for each $\mathbb{C} P^{N-1}$, after a Kähler transformation the Kähler potential can be rewritten as

$$
\begin{aligned}
K & =\sum_{I=1}^{k}\left[\pi v^{2}\left|z_{I}\right|^{2}+\frac{4 \pi}{g^{2}} \log \left|\vec{\phi}_{I}\right|^{2}\right]-\sum_{I \neq J}\left[\frac{2 \pi v^{2} N c_{e}^{2}}{m_{e}^{2}} K_{0}\left(m_{e}\left|z_{I}-z_{J}\right|\right)\right] \\
& -\sum_{I \neq J}\left[\frac{2 \pi v^{2} N c_{g}^{2}}{m_{g}^{2}}\left(N \frac{\left|\boldsymbol{\phi}_{I} \cdot \boldsymbol{\phi}_{J}^{\dagger}\right|^{2}}{\left|\boldsymbol{\phi}_{I}\right|^{2}\left|\boldsymbol{\phi}_{J}\right|^{2}}-1\right) K_{0}\left(m_{g}\left|z_{I}-z_{J}\right|\right)\right] .
\end{aligned}
$$

For the case $m_{e} \geq 2 m_{g}$, the second terms of Eq.(4.46) and Eq.(4.47) are incorrect since we have used Eq.(2.38) as an asymptotic behavior of $\psi_{e}$. They should be replaced with terms of order $e^{-2 m_{g}\left|z_{I}-z_{J}\right|}$. However, the dominant contribution to the interaction comes from the last term, which is of order $e^{-m_{g}\left|z-z_{0}\right|}$. Therefore we can say that the above results are correct even in the case of $m_{e} \geq 2 m_{g}$ if we neglect subleading contributions to the interaction.

\section{The point source formalism}

It has been shown that the interaction between well-separated Abelian vortices can be identified with that between composites of point-like scalar source and magnetic dipole [13, 39]. In [40] some of us used the point particle approximation for vortex-strings stretched between domain walls and found a good agreement with the direct calculation. 
In this section, we show that a non-Abelian vortex looks like a point particle in the following linear field theory

$$
\begin{aligned}
\mathcal{L}= & +\frac{1}{2}\left(\partial_{\mu} \Phi^{0} \partial^{\mu} \Phi^{0}-m_{e}^{2} \Phi^{0} \Phi^{0}\right)-\frac{1}{4 e^{2}}\left(f_{\mu \nu} f^{\mu \nu}-2 m_{e}^{2} w_{\mu} w^{\mu}\right) \\
& +\frac{1}{2}\left(\partial_{\mu} \Phi^{a} \partial^{\mu} \Phi^{a}-m_{g}^{2} \Phi^{a} \Phi^{a}\right)-\frac{1}{4 g^{2}}\left(F_{\mu \nu}^{a} F^{a \mu \nu}-2 m_{g}^{2} W_{\mu}^{a} W^{a \mu}\right) \\
& +\kappa^{0} \Phi^{0}+\kappa^{a} \Phi^{a}-\frac{1}{e^{2}} j^{0 \mu} w_{\mu}-\frac{1}{g^{2}} j^{a \mu} W_{\mu}^{a} .
\end{aligned}
$$

Here, $\Phi^{0}$ and $\Phi^{a}\left(a=1, \cdots, N^{2}-1\right)$ are scalar fields, $w_{\mu}$ and $W_{\mu}^{a}\left(a=1, \cdots, N^{2}-1\right)$ are the massive vector fields and $\kappa^{0}, \kappa^{a}, j_{\mu}^{0}, j_{\mu}^{a}$ are point-like sources corresponding to a non-Abelian vortex. Let us consider the following scalar source $\kappa \equiv \kappa^{0} t^{0}+\kappa^{a} t^{a}$

$$
\kappa=-\pi v\left[1-\frac{\left|\dot{z}_{0}\right|^{2}}{2}+\frac{1}{m_{g}^{2}}\left(\nabla_{t} \dot{\beta}^{i} \frac{\partial}{\partial \beta^{i}}+\overline{\nabla_{t} \dot{\beta}^{i}} \frac{\partial}{\partial \bar{\beta}^{i}}\right)\right]\left(c_{e} \mathbf{1}_{N}+c_{g} T_{\beta}\right) \delta^{2}\left(z-z_{0}\right),
$$

where $T_{\beta}$ is defined in Eq. (2.35) and $\nabla_{t} \dot{\beta}^{i}$ is defined by

$$
\nabla_{t} \dot{\beta}^{i} \equiv\left(\partial_{t}-\frac{2 \bar{\beta}^{j} \dot{\beta}^{j}}{1+\left|\beta^{j}\right|^{2}}\right) \dot{\beta}^{i}
$$

For the vector source $j_{\mu} \equiv j_{\mu}^{0} t^{0}+j_{\mu}^{a} t^{a}$, we assume the following form

$$
j^{\mu}=\partial_{\nu} X^{\mu \nu}
$$

where $X^{\mu \nu}$ is an anti-symmetric tensor. Note that this form of the vector current implies the conservation law $\partial_{\mu} j^{\mu}=0$. For the anti-symmetric tensor $X^{\mu \nu}$, let us consider the following form

$$
X^{\mu \nu}=-\pi\left[\epsilon^{\mu \nu \rho} J_{\rho}+\frac{1}{m_{g}^{2}}\left(\partial^{\mu} J^{\nu}-\partial^{\nu} J^{\mu}\right) i\left(\dot{\beta}^{i} \frac{\partial}{\partial \beta^{i}}-\dot{\bar{\beta}}^{i} \frac{\partial}{\partial \bar{\beta}^{i}}\right)\right]\left(c_{e} \mathbf{1}_{N}+c_{g} T_{\beta}\right) .
$$

where $J^{\mu}$ is the current of a point particle

$$
J^{\mu} \equiv \dot{x}_{0}^{\mu} \delta^{2}\left(z-z_{0}\right)
$$

Now we will see that a non-Abelian vortex, viewed from distance, looks like the above point source at least up to second order in the time-derivative. We can check this by comparing the field configuration for the point source and the asymptotic fields for a non-Abelian vortex derived in Appendix A. The equations of motion for the scalar fields $\Phi^{0}, \Phi^{a}$ and the vector fields $w_{\mu}$, $W_{\mu}$ are

$$
\begin{aligned}
\kappa^{0} & =\left(\partial_{\mu} \partial^{\mu}+m_{e}^{2}\right) \Phi^{0}, & \kappa^{a} & =\left(\partial_{\mu} \partial^{\mu}+m_{g}^{2}\right) \Phi^{a}, \\
j_{\nu}^{0} & =\left(\partial_{\mu} \partial^{\mu}+m_{e}^{2}\right) w_{\nu}, & j_{\nu}^{a} & =\left(\partial_{\mu} \partial^{\mu}+m_{g}^{2}\right) W_{\nu}^{a},
\end{aligned}
$$

where we have assumed that the vector fields satisfy the Lorenz gauge condition

$$
\partial_{\mu} w^{\mu}=\partial_{\mu} W^{a \mu}=0
$$


Operating $\partial^{\nu}$ on both sides of (5.8), we see that this gauge condition implies current conservation. The solution of the equations (5.7) and (5.8) can be obtained by using the Green's function expanded in terms of the time-derivative

$$
\begin{aligned}
\Phi^{0} & =\frac{1}{2 \pi} \int d^{2} z^{\prime}\left[K_{0}\left(m_{e}\left|z-z^{\prime}\right|\right)+\frac{1}{m_{e}^{2}} \Upsilon\left(m_{e}\left|z-z^{\prime}\right|\right) \partial_{t}^{2}+\mathcal{O}\left(\partial_{t}^{4}\right)\right] \kappa^{0}\left(t, z^{\prime}\right), \\
\Phi^{a} & =\frac{1}{2 \pi} \int d^{2} z^{\prime}\left[K_{0}\left(m_{g}\left|z-z^{\prime}\right|\right)+\frac{1}{m_{g}^{2}} \Upsilon\left(m_{g}\left|z-z^{\prime}\right|\right) \partial_{t}^{2}+\mathcal{O}\left(\partial_{t}^{4}\right)\right] \kappa^{a}\left(t, z^{\prime}\right), \\
w_{\mu} & =\frac{1}{2 \pi} \int d^{2} z^{\prime}\left[K_{0}\left(m_{e}\left|z-z^{\prime}\right|\right)+\frac{1}{m_{e}^{2}} \Upsilon\left(m_{e}\left|z-z^{\prime}\right|\right) \partial_{t}^{2}+\mathcal{O}\left(\partial_{t}^{4}\right)\right] j_{\mu}^{0}\left(t, z^{\prime}\right), \\
W_{\mu}^{a} & =\frac{1}{2 \pi} \int d^{2} z^{\prime}\left[K_{0}\left(m_{g}\left|z-z^{\prime}\right|\right)+\frac{1}{m_{g}^{2}} \Upsilon\left(m_{g}\left|z-z^{\prime}\right|\right) \partial_{t}^{2}+\mathcal{O}\left(\partial_{t}^{4}\right)\right] j_{\mu}^{a}\left(t, z^{\prime}\right) .
\end{aligned}
$$

where

$$
\Upsilon(s)=\frac{1}{2} s K_{0}^{\prime}(s)
$$

It is convenient to expand the sources in terms of the time-derivative

$$
\kappa=\kappa^{(0)}+\kappa^{(1)}+\kappa^{(2)}+\mathcal{O}\left(\partial_{t}^{3}\right), \quad j_{\mu}=j_{\mu}^{(0)}+j_{\mu}^{(1)}+j_{\mu}^{(2)}+\mathcal{O}\left(\partial_{t}^{3}\right) .
$$

Correspondingly, the scalar field $\Phi \equiv \Phi^{0} t^{0}+\Phi^{a} t^{a}$ and the vector field $W_{\mu}=w_{\mu} t^{0}+W_{\mu}^{a} t^{a}$ are expanded as

$$
\Phi=\Phi^{(0)}+\Phi^{(1)}+\Phi^{(2)}+\mathcal{O}\left(\partial_{t}^{3}\right), \quad W_{\mu}=W_{\mu}^{(0)}+W_{\mu}^{(1)}+W_{\mu}^{(2)}+\mathcal{O}\left(\partial_{t}^{3}\right) .
$$

Let us check that the field configuration for the point source and that for a non-Abelian vortex order by order. Note that odd (even) order equations of motion for $\Phi^{(n)}, w_{\bar{z}}^{(n)}$ and $W_{\bar{z}}^{(n)}\left(w_{0}^{(n)}\right.$ and $\left.W_{0}^{(n)}\right)$ are trivial due to time-reversal symmetry. First, we consider the leading terms $\Phi^{(0)}$ and $W_{\mu}^{(0)}$ corresponding to the static configuration. The leading order terms of the sources are given by

$$
\kappa^{(0)}=-\pi v\left(c_{e} \mathbf{1}_{N}+c_{g} T_{\beta}\right) \delta^{2}\left(z-z_{0}\right), \quad j_{\bar{z}}^{(0)}=-\pi i\left(c_{e} \mathbf{1}_{N}+c_{g} T_{\beta}\right) \bar{\partial} \delta^{2}\left(z-z_{0}\right) .
$$

Note that $j_{0}$ has no term without time-derivative. Evaluating (5.10), (5.11), (5.12) and (5.13), we obtain the following solutions for the static source

$$
\Phi^{(0)}=-\frac{v}{2} \Psi, \quad W_{\bar{z}}^{(0)}=-\frac{i}{2} \bar{\partial} \Psi
$$

where the function $\Psi$ is given by

$$
\Psi \equiv c_{e} \mathbf{1}_{N} K_{0}\left(m_{e}\left|z-z_{0}\right|\right)+c_{g} T_{\beta} K_{0}\left(m_{e}\left|z-z_{0}\right|\right) .
$$

These solutions agree with the asymptotic forms of the static single vortex solution (A.6) and (A.7) in a singular gauge. Next, let us consider the first order contribution which are contained only in $j_{0}$

$$
j_{0}^{(1)}=-\pi i\left[\dot{z}_{0} \frac{\partial}{\partial z_{0}}-\dot{\bar{z}}_{0} \frac{\partial}{\partial \bar{z}_{0}}+\frac{4}{m_{g}^{2}} \frac{\partial}{\partial z_{0}} \frac{\partial}{\partial \bar{z}_{0}}\left(\dot{\beta}^{i} \frac{\partial}{\partial \beta^{i}}-\dot{\bar{\beta}}^{i} \frac{\partial}{\partial \bar{\beta}^{i}}\right)\right]\left(c_{e} \mathbf{1}_{N}+c_{g} T_{\beta}\right) \delta^{2}\left(z-z_{0}\right) .
$$


By using (5.12), (15.13) and $\left(4 \partial \bar{\partial}-m^{2}\right) K_{0}\left(m\left|z-z_{0}\right|\right)=0\left(z \neq z_{0}\right)$, we obtain

$$
W_{0}^{(1)}=\frac{i}{2}\left[\dot{z}_{0} \frac{\partial}{\partial z_{0}}-\dot{\bar{z}}_{0} \frac{\partial}{\partial \bar{z}_{0}}+\dot{\beta}^{i} \frac{\partial}{\partial \beta^{i}}-\dot{\bar{\beta}}^{i} \frac{\partial}{\partial \bar{\beta}^{i}}\right] \Psi .
$$

The second order terms in the scalar source $\kappa$ and the current $j_{\bar{z}}$ are given by

$$
\begin{aligned}
\kappa^{(2)}= & \pi v\left[\frac{\left|\dot{z}_{0}\right|^{2}}{2}-\frac{1}{m_{g}^{2}}\left(\nabla_{t} \dot{\beta}^{i} \frac{\partial}{\partial \beta^{i}}+\overline{\nabla_{t} \dot{\beta}^{i}} \frac{\partial}{\partial \bar{\beta}^{i}}\right)\right] \delta^{2}\left(z-z_{0}\right)\left(c_{e} \mathbf{1}_{N}+c_{g} T_{\beta}\right), \\
j_{\bar{z}}^{(2)}= & -\frac{\pi i}{2}\left(\ddot{z}_{0}+\dot{z}_{0} \frac{\partial}{\partial z_{0}}+\dot{\bar{z}}_{0} \frac{\partial}{\partial \bar{z}_{0}}\right) \delta^{2}\left(z-z_{0}\right)\left(c_{e} \mathbf{1}_{N}+c_{g} T_{\beta}\right) \\
& -\frac{2 \pi}{m_{g}^{2}} i\left(\dot{\beta}^{i} \frac{\partial}{\partial \beta^{i}}-\dot{\bar{\beta}}^{i} \frac{\partial}{\partial \bar{\beta}^{i}}\right) \dot{z}_{0} \frac{\partial}{\partial z_{0}} \frac{\partial}{\partial \bar{z}_{0}} \delta^{2}\left(z-z_{0}\right)\left(c_{e} \mathbf{1}_{N}+c_{g} T_{\beta}\right) \\
& -\frac{\pi i}{m_{g}^{2}}\left(\nabla_{t} \dot{\beta}^{i} \frac{\partial}{\partial \beta^{i}}-\nabla_{t} \dot{\bar{\beta}}^{i} \frac{\partial}{\partial \bar{\beta}^{i}}\right) \frac{\partial}{\partial \bar{z}_{0}} \delta^{2}\left(z-z_{0}\right)\left(c_{e} \mathbf{1}_{N}+c_{g} T_{\beta}\right) .
\end{aligned}
$$

By evaluating the integral (5.10), (5.11), (5.12) and (5.13), we obtain the following solutions

$$
\begin{aligned}
\Phi^{(2)}= & \frac{1}{8} v\left[\ddot{z}_{0}\left(\bar{z}-\bar{z}_{0}\right)+\ddot{\bar{z}}_{0}\left(z-z_{0}\right)\right] \Psi \\
& -\frac{1}{8} v\left[\dot{z}_{0}\left(\bar{z}-\bar{z}_{0}\right)+\dot{\bar{z}}_{0}\left(z-z_{0}\right)\right]\left(\dot{z}_{0} \partial+\dot{\bar{z}}_{0} \bar{\partial}\right) \Psi \\
& +\frac{1}{4} v\left[\dot{z}_{0}\left(\bar{z}-\bar{z}_{0}\right)+\dot{\bar{z}}_{0}\left(z-z_{0}\right)\right]\left(\dot{\beta}^{i} \frac{\partial}{\partial \beta^{i}}+\dot{\bar{\beta}}^{i} \frac{\partial}{\partial \bar{\beta}^{i}}\right) \Psi \\
& -\frac{1}{2 m_{g}^{2}} v\left(\nabla_{t} \dot{\beta}^{i} \frac{\partial}{\partial \beta^{i}}+\overline{\nabla_{t} \dot{\beta}^{i}} \frac{\partial}{\partial \bar{\beta}^{i}}\right) \Psi \\
& -\frac{1}{2 m_{g}^{2}} v c_{g} \Upsilon\left(m_{g}\left|z-z_{0}\right|\right) \partial_{t}^{2} T_{\beta}, \\
W_{\bar{z}}^{(2)}= & \frac{i}{8}\left[\ddot{z}_{0}\left(\bar{z}-\bar{z}_{0}\right)+\ddot{\bar{z}}_{0}\left(z-z_{0}\right)\right] \bar{\partial} \Psi \\
& -\frac{i}{8}\left[\dot{z}_{0}\left(\bar{z}-\bar{z}_{0}\right)+\dot{\bar{z}}_{0}\left(z-z_{0}\right)\right]\left(\dot{z}_{0} \partial+\dot{\bar{z}}_{0} \bar{\partial}\right) \bar{\partial} \Psi \\
& -\frac{i}{8}\left[\ddot{z}_{0}-\dot{z}_{0}\left(\dot{z}_{0} \partial-\dot{\bar{z}}_{0} \bar{\partial}\right)\right] \Psi \\
& +\frac{i}{4}\left[\dot{z}_{0}\left(\bar{z}-\bar{z}_{0}\right)+\dot{\bar{z}}_{0}\left(z-z_{0}\right)\right]\left(\dot{\beta}^{i} \frac{\partial}{\partial \beta^{i}}+\dot{\bar{\beta}}^{i} \frac{\partial}{\partial \bar{\beta}^{i}}\right) \bar{\partial} \Psi \\
& -\frac{i}{m_{g}^{2}} \dot{z}_{0}\left(\dot{\beta}^{i} \frac{\partial}{\partial \beta^{i}}-\dot{\bar{\beta}}^{i} \frac{\partial}{\partial \bar{\beta}^{i}}\right) \partial \bar{\partial} \Psi \\
& +\frac{i}{2 m_{g}^{2}}\left(\nabla_{t} \dot{\beta}^{i} \frac{\partial}{\partial \beta^{i}}-\frac{\partial}{\nabla_{t} \dot{\beta}^{i}} \frac{\partial}{\partial \bar{\beta}^{i}}\right) \bar{\partial} \Psi \\
& -\frac{i}{2 m_{g}^{2}} c_{g} \bar{\partial} \Upsilon\left(m_{g}\left|z-z_{J}\right|\right) \partial_{t}^{2} T_{\beta}, \\
&
\end{aligned}
$$

As shown in the Appendix A, the solutions (5.21), (5.24) and (5.25) agree with those obtained by using the method of derivative expansion with a moving vortex background. 
Let us rederive the effective Lagrangian for the non-Abelian vortices Eq. (4.45) from the point particle formalism. The effective Lagrangian for the point particles are given by

$$
L_{\mathrm{eff}}=\sum_{I=1}^{k}\left(\pi v\left|\dot{z}_{I}\right|^{2}+\frac{4 \pi}{g^{2}} \dot{\beta}^{i} \dot{\bar{\beta}}^{j} \frac{\partial^{2}}{\partial \beta^{i} \bar{\beta}^{j}} \log \left(1+\left|\beta^{i}\right|^{2}\right)\right)+\sum_{I>J} L_{(I, J)} .
$$

Here, $L_{(I, J)}$ is the interaction Lagrangian between $I$-th and $J$-th particles

$$
L_{(I, J)}=\int d^{2} x\left(\kappa_{I}^{0} \Phi_{J}^{0}+\kappa_{I}^{a} \Phi_{J}^{a}-\frac{1}{e^{2}} j_{I}^{0 \mu} w_{\mu J}-\frac{1}{g^{2}} j_{I}^{a \mu} W_{\mu J}^{a}\right)
$$

$\kappa_{I}^{0}, \kappa_{I}^{a}, j_{\mu I}^{0}$ and $j_{\mu I}^{a}$ are the point-like source with $z_{0}=z_{I}, \beta^{i}=\beta_{I}^{i}$ and $\Phi_{J}^{0}, \Phi_{J}^{a}, w_{\mu J}$ and $W_{\mu J}$ are the fields induced by the point-like source with $z_{0}=z_{J}, \beta^{i}=\beta_{J}^{i}$. First, let us consider static interaction

$$
L_{(I, J)}^{(0)}=\frac{1}{2} \int d^{2} x\left[\kappa_{I}^{0(0)} \Phi_{J}^{0(0)}+\frac{4}{e^{2}} j_{z I}^{0(0)} w_{\bar{z} J}^{(0)}+\kappa_{I}^{a(0)} \Phi_{J}^{a(0)}+\frac{4}{g^{2}} j_{z I}^{a(0)} W_{\bar{z} J}^{a(0)}+(\text { c.c. })\right] .
$$

Since the static sources $\kappa_{I}^{(0)}$ and $j_{z I}^{(0)}$ are given by

$$
\kappa_{I}^{(0)}=-\pi v\left(c_{e} \mathbf{1}_{N}+c_{g} T_{\beta_{I}}\right) \delta^{2}\left(z-z_{I}\right), \quad j_{z I}^{(0)}=\pi i\left(c_{e} \mathbf{1}_{N}+c_{g} T_{\beta_{I}}\right) \partial \delta^{2}\left(z-z_{I}\right),
$$

the static interaction Lagrangian reduces to

$$
L_{(I, J)}^{(0)}=-\pi v \operatorname{tr}\left[\left(\Phi_{J}^{0(0)} t^{0}+\frac{4 v}{m_{e}^{2}} i \partial w_{\bar{z} J}^{(0)} t^{0}+\Phi_{J}^{a(0)} t^{a}+\frac{4 v}{m_{g}^{2}} i \partial W_{\bar{z} J}^{a(0)} t^{a}\right)\left(c_{e} \mathbf{1}_{N}+c_{g} T_{\beta_{I}}\right)\right]_{z=z_{I}} .
$$

We can show by using Eq. (5.18) and $\left(4 \partial \bar{\partial}-m^{2}\right) K_{0}\left(m\left|z-z_{0}\right|\right)=0$ that

$$
\left(\Phi_{J}^{0(0)} t^{0}+\frac{4 v}{m_{e}^{2}} i \partial W_{\bar{z} J}^{0(0)} t^{0}\right)=\left(\Phi_{J}^{a(0)} t^{a}+\frac{4 v}{m_{g}^{2}} i \partial W_{\bar{z} J}^{a(0)} t^{a}\right)=0 .
$$

Therefore, the static interaction vanishes as expected from the BPS property. The leading order terms in the interaction Lagrangian are second order in the time-derivative

$$
\begin{aligned}
L_{(I, J)}^{(2)}=\int d^{2} x & {\left[\operatorname{Re}\left(\kappa_{I}^{0(0)} \Phi_{J}^{0(2)}+\frac{4}{e^{2}} j_{z I}^{0(0)} W_{\bar{z} J}^{0(2)}+\kappa_{I}^{a(0)} \Phi_{J}^{a(2)}+\frac{4}{g^{2}} j_{z I}^{a(0)} W_{\bar{z} J}^{a(2)}\right)+(I \leftrightarrow J)\right.} \\
& -\partial_{t} \Phi_{I}^{0(0)} \partial_{t} \Phi_{J}^{0(0)}-\partial_{t} \Phi_{I}^{a(0)} \partial_{t} \Phi_{J}^{a(0)}-\frac{4}{e^{2}} \partial_{t} W_{z I}^{0(0)} \partial_{t} W_{\bar{z} J}^{0(0)}-\frac{4}{g^{2}} \partial_{t} W_{z I}^{a(0)} \partial_{t} W_{\bar{z} J}^{a(0)} \\
& \left.-\frac{1}{e^{2}} j_{0 I}^{0(1)} W_{0 J}^{0(1)}-\frac{1}{g^{2}} j_{0 I}^{a(1)} W_{0 J}^{a(1)}\right]
\end{aligned}
$$

Here, we have ignored total time derivatives and used

$$
\int d^{2} x \kappa_{I}^{0(2)} \Phi_{J}^{0(0)}=\int d^{2} x\left[\partial_{t} \partial_{t} \Phi_{I}^{0(0)} \Phi_{J}^{0(0)}+\Phi_{I}^{0(2)} \kappa_{J}^{0(0)}\right]
$$


and similar identities for the other fields. To calculate the interaction Lagrangian $L_{(I, J)}^{(2)}$, it is convenient to decompose it as

$$
\begin{aligned}
\mathcal{A}_{e} & \equiv \int d^{2} x \operatorname{Re}\left(\kappa_{I}^{0(0)} \Phi_{J}^{0(2)}+\frac{4}{e^{2}} j_{z I}^{0(0)} W_{\bar{z} J}^{0(2)}\right), \\
\mathcal{A}_{g} & \equiv \int d^{2} x \operatorname{Re}\left(\kappa_{I}^{a(0)} \Phi_{J}^{a(2)}+\frac{4}{g^{2}} j_{z I}^{a(0)} W_{\bar{z} J}^{a(2)}\right), \\
\mathcal{B}_{1} & \equiv \int d^{2} x\left(\partial_{t} \Phi_{I}^{0(0)} \partial_{t} \Phi_{J}^{0(0)}+\partial_{t} \Phi_{I}^{a(0)} \partial_{t} \Phi_{J}^{a(0)}\right), \\
\mathcal{B}_{2} & \equiv \int d^{2} x\left(\frac{4}{e^{2}} \partial_{t} W_{z I}^{0(0)} \partial_{t} W_{\bar{z} J}^{0(0)}+\frac{4}{g^{2}} \partial_{t} W_{z I}^{a(0)} \partial_{t} W_{\bar{z} J}^{a(0)}\right), \\
\mathcal{C} & \equiv \int d^{2} x\left(\frac{1}{e^{2}} j_{0 I}^{0(1)} W_{0 J}^{0(1)}+\frac{1}{e^{2}} j_{0 I}^{a(1)} W_{0 J}^{a(1)}\right) .
\end{aligned}
$$

By using the explicit form of the static sources Eq. (5.29) and the solutions (5.24) and (5.25), we obtain

$$
\begin{aligned}
\mathcal{A}_{e}+\mathcal{A}_{g} & =-2 \pi v \operatorname{Re}\left[c_{e} \operatorname{Tr}\left(\Phi_{J}^{0(2)} t^{0}+\frac{4 v}{m_{e}^{2}} i \partial W_{\bar{z} J}^{0(2)} t^{0}\right)+c_{g} \operatorname{Tr}\left(\Phi_{J}^{a(2)} t^{a}+\frac{4 v}{m_{e}^{2}} i \partial W_{\bar{z} J}^{a(2)} t^{a}\right)\right]_{z=z_{I}} \\
& =-\delta_{J} \delta_{J}^{\dagger}\left[\frac{2 \pi c_{e}^{2}}{e^{2}} N K_{0}\left(m_{e}\left|z_{I}-z_{J}\right|\right)+\frac{2 \pi c_{e}^{2}}{g^{2}} \operatorname{Tr}\left[T_{\beta_{I}} T_{\beta_{J}}\right] K_{0}\left(m_{g}\left|z_{I}-z_{J}\right|\right)\right]
\end{aligned}
$$

where we have defined the differential operators $\delta_{J}, \delta_{J}^{\dagger}$ by

$$
\delta_{J} \equiv \dot{z}_{J} \frac{\partial}{\partial z_{J}}+\dot{\beta}_{J}^{i} \frac{\partial}{\partial \beta_{J}^{i}}, \quad \delta_{J}^{\dagger} \equiv \dot{\bar{z}}_{J} \frac{\partial}{\partial \bar{z}_{J}}+\dot{\bar{\beta}}_{J}^{i} \frac{\partial}{\partial \bar{\beta}_{J}^{i}} .
$$

By using the identity

$$
\int d^{2} x K_{0}\left(m\left|z-z_{I}\right|\right) K_{0}\left(m\left|z-z_{J}\right|\right)=-\frac{2 \pi}{m^{2}} \Upsilon\left(m\left|z_{I}-z_{J}\right|\right)
$$

with $\Upsilon$ in Eq.(5.14), we can calculate $\mathcal{B}_{1}$ and $\mathcal{B}_{2}$ as

$$
\begin{aligned}
\mathcal{B}_{1}= & \left(\delta_{I}+\delta_{I}^{\dagger}\right)\left(\delta_{J}+\delta_{J}^{\dagger}\right)\left[-\frac{\pi c_{e}^{2}}{e^{2}} N \Upsilon\left(m_{e}\left|z_{I}-z_{J}\right|\right)-\frac{\pi c_{g}^{2}}{g^{2}} \operatorname{Tr}\left[T_{\beta_{I}} T_{\beta_{J}}\right] \Upsilon\left(m_{g}\left|z_{I}-z_{J}\right|\right)\right], \\
\mathcal{B}_{2}= & \left(\delta_{I}+\delta_{I}^{\dagger}\right)\left(\delta_{J}+\delta_{J}^{\dagger}\right) \\
& \times \frac{\partial}{\partial z_{I}} \frac{\partial}{\partial \bar{z}_{J}}\left[-\frac{4 \pi c_{e}^{2}}{m_{e}^{2} e^{2}} N \Upsilon\left(m_{e}\left|z_{I}-z_{J}\right|\right)-\frac{4 \pi c_{g}^{2}}{m_{g}^{2} g^{2}} \operatorname{Tr}\left[T_{\beta_{I}} T_{\beta_{J}}\right] \Upsilon\left(m_{g}\left|z_{I}-z_{J}\right|\right)\right]
\end{aligned}
$$

Therefore, we find that

$$
\mathcal{B}_{1}+\mathcal{B}_{2}=\left(\delta_{I}+\delta_{I}^{\dagger}\right)\left(\delta_{J}+\delta_{J}^{\dagger}\right)\left[\frac{\pi c_{e}^{2}}{e^{2}} N K_{0}\left(m_{e}\left|z_{I}-z_{J}\right|\right)+\frac{\pi c_{g}^{2}}{g^{2}} \operatorname{Tr}\left[T_{\beta_{I}} T_{\beta_{J}}\right] K_{0}\left(m_{g}\left|z_{I}-z_{J}\right|\right)\right]
$$

where we have used $\left(-4 \partial \bar{\partial}+m^{2}\right) \Upsilon\left(m\left|z-z_{0}\right|\right)=-m^{2} K_{0}\left(m\left|z-z_{0}\right|\right)$. From (15.20) and (5.21), we obtain

$$
\mathcal{C}=-\left(\delta_{I}-\delta_{I}^{\dagger}\right)\left(\delta_{J}-\delta_{J}^{\dagger}\right)\left[\frac{\pi c_{e}^{2}}{e^{2}} N K_{0}\left(m_{e}\left|z_{I}-z_{J}\right|\right)+\frac{\pi c_{g}^{2}}{g^{2}} \operatorname{Tr}\left[T_{\beta_{I}} T_{\beta_{J}}\right] K_{0}\left(m_{g}\left|z_{I}-z_{J}\right|\right)\right] .
$$


Therefore, the interaction Lagrangian is given by

$$
\begin{aligned}
L_{(I, J)} & =\left[\mathcal{A}_{e}+\mathcal{A}_{g}+(I \leftrightarrow J)\right]-\left[\mathcal{B}_{1}+\mathcal{B}_{2}+\mathcal{C}\right] \\
& =-\left(\delta_{I}+\delta_{J}\right)\left(\delta_{I}^{\dagger}+\delta_{J}^{\dagger}\right)\left[\frac{2 \pi c_{e}^{2}}{e^{2}} N K_{0}\left(m_{e}\left|z_{I}-z_{J}\right|\right)+\frac{2 \pi c_{g}^{2}}{g^{2}} \operatorname{Tr}\left[T_{\beta_{I}} T_{\beta_{J}}\right] K_{0}\left(m_{g}\left|z_{I}-z_{J}\right|\right)\right] .
\end{aligned}
$$

This interaction Lagrangian agrees with that in the effective Lagrangian Eq. (4.45).

\section{Conclusions and Discussions}

By exploiting the local nature of non-Abelian vortices for $N_{\mathrm{C}}=N_{\mathrm{F}}$, we have extended the Samols' formula of for the metric on the moduli space to the non-Abelian $U(N)$ case. This fact has enabled us to construct the explicit metric (4.45) and its Kähler potential (4.46) on the moduli space of well-separated non-Abelian vortices. We have also derived the metric using an appropriate point-particle approximation for non-Abelian vortices.

In this paper we have studied local vortices in $U(N)$ gauge theory with the same number of fundamental Higgs fields as the number $N$ of colors. When the flavor number is greater than the color number, the vortices are instead called semi-local [32]. A typical property of semilocal vortices is that they have a size modulus which is in general non-normalizable. In the case of a semi-local non-Abelian vortex, its orientational moduli are also non-normalizable [33] unless its size modulus vanishes[29]. The (non-)normalizability of zero modes was completely classified in [29] for arbitrary number of vortices with arbitrary moduli. Since wave functions for non-normalizable moduli are divergent in infinite space, we can have a metric only for the normalizable moduli. The most direct way to extend the present work to the case of semi-local non-Abelian vortices would be the calculation of such metric through the generalization of Samols' formula. However, the metric of well-separated semi-local vortices should be well-approximated by that of lumps [41], since the asymptotic behavior in this case is efficiently described by lumps rather than local vortices. Therefore one should be able to work out the metric without the generalization of Samols' formula [42].

In order to address the metric problem in different gauge groups, such as $S O$ and $S p$, one must take into account the observation that non-Abelian vortices are generically semi-local. This means that our result can be immediately generalized only to the particular sector of their moduli space where vortices happen to be local, while for the full space a supplementary work of the type mentioned in the previous paragraphs will be needed. Non-Abelian vortices with gauge group $G \times U(1)$ with arbitrary simple group $G$ were constructed in [34]. It was found for instance that the moduli spaces of single local vortices in $G=S O(2 N), U S p(2 N)$ gauge theories are Hermitian symmetric spaces $S O(2 N) / U(N)$ and $U S p(2 N) / U(N)$, respectively. The moduli space of nonAbelian vortices in $S O$ and $U S p$ gauge theories were further studied in [35, 36, 37, 38].

Other possible directions are:

i) to obtain the generalization of the Samols' formula to geometries other than $\mathbb{C}$, for instance, a cylinder [43], a torus [44], Riemann surfaces [45] or a hyperbolic space [15, 14, 46], 
ii) the inclusion of a Chern-Simons term [47],

iii) the generalization to the non-BPS case where a potential term will be induced on the moduli space [48].

\section{Acknowledgments}

We would like to thank Minoru Eto for discussion in the early stage of this work. G. M. acknowledges support from "A. Della Riccia" Foundation and Japan Society for Promotion of Science. The work of M.N. and of N.S are supported in part by Grant-in-Aid for Scientific Research No. 20740141 (M.N.), No. 21540279 (N.S.) and No. 21244036 (N.S.) from the Ministry of Education, Culture, Sports, Science and Technology-Japan.

\section{A Derivative expansion}

In this section, we will see that the solutions (5.24) and (5.25) agree with the asymptotic fields for the vortex configuration by using the derivative expansion. It is convenient to use a singular gauge by performing the following singular gauge transformations $\mathcal{U}_{e} \in U(1)$ and $\mathcal{U}_{g} \in S U(N)$

$$
\mathcal{U}_{e}=\left(\frac{\bar{z}-\bar{z}_{0}}{\left|z-z_{0}\right|}\right)^{\frac{1}{N} \mathbf{1}_{N}}, \quad \mathcal{U}_{g}=U^{\dagger}\left(\frac{\bar{z}-\bar{z}_{0}}{\left|z-z_{0}\right|}\right)^{\frac{1}{N} T} U
$$

Then, the solutions for a single vortex in a non-singular gauge Eq. (2.31) become

$$
S_{e}=v^{-1}\left(z-z_{0}\right)^{\frac{1}{N} \mathbf{1}_{N}} e^{\frac{1}{2} \hat{\psi}_{e} \mathbf{1}_{N}}, \quad S_{g}=V\left(z-z_{0}\right)^{\frac{1}{N} T} e^{\frac{1}{2} \hat{\psi}_{g} T} U
$$

where $\hat{\psi}_{e}$ and $\hat{\psi}_{g}$ are defined by

$$
\begin{aligned}
& \hat{\psi}_{e} \equiv \psi_{e}-\frac{1}{N} \log \left|z-z_{0}\right|^{2} \approx c_{e} K_{0}\left(m_{e}\left|z-z_{0}\right|\right)+\cdots \\
& \hat{\psi}_{g} \equiv \psi_{g}-\frac{1}{N} \log \left|z-z_{0}\right|^{2} \approx c_{g} K_{0}\left(m_{g}\left|z-z_{0}\right|\right)+\cdots
\end{aligned}
$$

Note that $S_{e} S_{g}$ is single-valued whereas each of $S_{e}$ and $S_{g}$ is multi-valued. Correspondingly, the solutions for the Higgs fields and the gauge fields (2.32), (2.33), (2.34) become

$$
H=v e^{-\frac{1}{2}\left(\hat{\psi}_{e} \mathbf{1}_{N}+\hat{\psi}_{g} T_{\beta}\right)}, \quad w_{\bar{z}} t^{0}+W_{\bar{z}}^{a} t^{a}=-\frac{i}{2} \bar{\partial}\left(\hat{\psi}_{e} \mathbf{1}_{N}+\hat{\psi}_{g} T_{\beta}\right),
$$

where $T_{\beta}$ is the matrix defined in Eq. (2.35). We focus on the asymptotic form of the single vortex solution in the singular gauge

$$
\begin{aligned}
H-v \mathbf{1}_{N} & =-\frac{1}{2} v c_{e} K_{0}\left(m_{e}\left|z-z_{0}\right|\right) \mathbf{1}_{N}-\frac{1}{2} v c_{g} K_{0}\left(m_{g}\left|z-z_{0}\right|\right) T_{\beta}+\cdots \\
w_{\bar{z}} t^{0}+W_{\bar{z}}^{a} t^{a} & =-\frac{i}{2} c_{e} \bar{\partial} K_{0}\left(m_{e}\left|z-z_{0}\right|\right) \mathbf{1}_{N}-\frac{i}{2} c_{g} \bar{\partial} K_{0}\left(m_{g}\left|z-z_{0}\right|\right) T_{\beta}+\cdots
\end{aligned}
$$


Identifying the scalar fields $\Phi \equiv \Phi^{0} t^{0}+\Phi^{a} t^{a}$ with $H-v \mathbf{1}_{N}$ in the singular gauge, we find that the terms without time-derivative in (5.18) agree with the static solutions (A.6) and (A.7). To check that the terms with time-derivative are also correct, let us consider a time-dependent background configuration by promoting the moduli parameters to time dependent dynamical valuables. The time-dependence induces the fluctuations around the background configuration, so we expand the fields with respect to the number of the time-derivatives

$$
H(t)=H^{\mathrm{BPS}}\left(z_{0}(t), \beta^{i}(t)\right)+H^{(1)}(t)+H^{(2)}(t)+\cdots,
$$

and similarly for the vector fields $w_{\mu}$ and $W_{\mu}^{a}$. As a background solution, let us consider a single BPS vortex configuration satisfying the following BPS equations

$$
\mathcal{D}_{\bar{z}} H=0, \quad \frac{4}{e^{2}} i f_{z \bar{z}} t^{0}+\frac{4}{g^{2}} i F_{z \bar{z}}^{a} t^{a}+H H^{\dagger}-v^{2} \mathbf{1}_{N}=0 .
$$

If the position moduli $z_{0}$ and the orientation $\beta^{i}$ are constant, this background configuration satisfies the equations of motion

$$
\begin{aligned}
0 & =\mathcal{D}_{\mu} \mathcal{D}^{\mu} H+e^{2}\left(H_{A}^{\dagger} t^{0} H_{A}-\xi\right) t^{0} H+g^{2}\left(H_{A}^{\dagger} t^{a} H_{A}\right) t^{a} H \\
0 & =\mathcal{D}^{\mu}\left(\frac{2}{e^{2}} f_{\mu \nu} t^{0}+\frac{2}{g^{2}} F_{\mu \nu}^{a} t^{a}\right)+i\left[H\left(\mathcal{D}_{\nu} H\right)^{\dagger}-\left(\mathcal{D}_{\nu} H\right) H^{\dagger}\right]
\end{aligned}
$$

If we give weak time dependences to the moduli parameters

$$
z_{0} \rightarrow z_{0}(t), \quad \beta^{i} \rightarrow \beta^{i}(t)
$$

Eq. (A.10) and Eq. (A.11) become equations of motion for the fluctuations, which can be solve order-by-order. Note that odd (even) order equations of motion for $H^{(n)}, w_{\bar{z}}^{(n)}$ and $W_{\bar{z}}^{(n)}\left(w_{0}^{(n)}\right.$ and $W_{0}^{(n)}$ ) are trivial due to the time-reversal symmetry. The first order term in the gauge fields $w_{0}$ and $W_{0}^{a}$ are determined from the Gauss' law equation

$$
\left.0=-\mathcal{D}_{z}\left(\frac{4}{e^{2}} f_{\bar{z} 0} t^{0}+\frac{4}{g^{2}} F_{\bar{z} 0}^{a} t^{a}\right)-i\left(\mathcal{D}_{0} H\right) H^{\dagger}+\text { (h.c. }\right) .
$$

This equation can be solved as

$$
w_{0}^{(1)} t^{0}=i\left(\delta_{t} S_{e}^{\dagger} S_{e}^{\dagger-1}-S_{e}^{-1} \delta_{t}^{\dagger} S_{e}\right), \quad W_{0}^{a(1)} t^{a}=i\left(\delta_{t} S_{g}^{\dagger} S_{g}^{\dagger-1}-S_{g}^{-1} \delta_{t}^{\dagger} S_{g}\right)
$$

By using the asymptotic forms (A.3) and (A.4), we can check that Eq. (5.21) agrees with the asymptotic forms of $w_{0}$ and $W_{0}$.

The asymptotic behavior of the second order fluctuations are determined from the linearized equations of motion

$$
\begin{aligned}
-\frac{1}{4} \mathcal{D}_{0} \mathcal{D}_{0} H & =i \mathcal{D}_{z} \mathcal{H}+\frac{1}{2}\left(\mathcal{W}_{e}+\mathcal{W}_{e}^{\dagger}+\mathcal{W}_{g}+\mathcal{W}_{g}^{\dagger}\right) H \\
-\mathcal{D}_{0}\left[\frac{1}{e^{2}} f_{0 \bar{z}} t^{0}+\frac{1}{g^{2}} F_{0 \bar{z}}^{a} t^{a}\right] & =i \mathcal{D}_{\bar{z}}\left[\frac{2}{e^{2}}\left(\mathcal{W}_{e}+\mathcal{W}_{e}^{\dagger}\right)+\frac{2}{g^{2}}\left(\mathcal{W}_{g}+\mathcal{W}_{g}^{\dagger}\right)\right]-\mathcal{H} H^{\dagger}
\end{aligned}
$$


Here, we have defined $\mathcal{H}, \mathcal{W}_{e}$ and $\mathcal{W}_{g}$ by

$$
\begin{aligned}
\mathcal{H} & \equiv i \mathcal{D}_{\bar{z}} H^{(2)}-\left(w_{\bar{z}}^{(2)} t^{0}+W_{\bar{z}}^{a(2)} t^{a}\right) H, \\
\mathcal{W}_{e} & \equiv i \mathcal{D}_{z}\left(w_{\bar{z}}^{(2)} t^{0}\right)+\frac{e^{2}}{2} \operatorname{Tr}\left(H^{(2)} H^{\dagger} t^{0}\right) t^{0}, \\
\mathcal{W}_{g} & \equiv i \mathcal{D}_{z}\left(W_{\bar{z}}^{a(2)} t^{a}\right)+\frac{g^{2}}{2} \operatorname{Tr}\left(H^{(2)} H^{\dagger} t^{a}\right) t^{a} .
\end{aligned}
$$

Note that the linearized equations of motion have the physical zero mode associated with the moduli parameters and the gauge zero modes corresponding to the gauge transformation acting on the fluctuations. The gauge transformation acts of the fluctuation fields as

$$
H^{(2)} \rightarrow H^{(2)}+i A^{(2)} H, \quad w_{\bar{z}}^{(2)} t^{0}+W_{\bar{z}}^{a(2)} t^{a} \rightarrow w_{\bar{z}}^{(2)} t^{0}+W_{\bar{z}}^{a(2)} t^{a}-\mathcal{D}_{\bar{z}} A^{(2)},
$$

where $A^{(2)}$ is an arbitrary hermitian matrix which is of second order in the time-derivative. To solve the equations (A.15) and (A.16), it is convenient to take the following gauge for the fluctuations

$$
\mathcal{W}_{e}=\mathcal{W}_{e}^{\dagger}, \quad \mathcal{W}_{g}=\mathcal{W}_{g}^{\dagger}
$$

Then, the equations (A.15) and (A.16) can be solved with respect to $\mathcal{H}, \mathcal{W}_{e}$ and $\mathcal{W}_{g}$ as

$$
\begin{gathered}
\mathcal{W}_{e}=-\frac{1}{4} S_{e}^{-1} S_{g}^{-1}\left[\dot{\phi}^{i} \dot{\bar{\phi}}^{j} \frac{\partial}{\partial \bar{\phi}^{j}}\left(\Omega_{e} \frac{\partial}{\partial \phi^{i}} \Omega_{e}^{-1}\right)\right] S_{e} S_{g} \\
\mathcal{W}_{g}=-\frac{1}{4} S_{e}^{-1} S_{g}^{-1}\left[\dot{\phi}^{i} \dot{\bar{\phi}}^{j} \frac{\partial}{\partial \bar{\phi}^{j}}\left(\Omega_{g} \frac{\partial}{\partial \phi^{i}} \Omega_{g}^{-1}\right)\right] S_{e} S_{g} \\
\mathcal{H}=S_{e}^{\dagger} S_{g}^{\dagger}\left[\left(\ddot{\phi}^{i} \frac{\partial}{\partial \phi^{i}}+\dot{\phi}^{i} \dot{\phi}^{j} \frac{\partial}{\partial \phi^{i}} \frac{\partial}{\partial \phi^{j}}\right)\left(\frac{1}{e^{2}} \bar{\partial} \Omega_{e}^{-1} \Omega_{e}+\frac{1}{g^{2}} \bar{\partial} \Omega_{g}^{-1} \Omega_{g}\right)\right] H_{0}^{\dagger-1} .
\end{gathered}
$$

The fluctuations $H^{(2)}, w_{\bar{z}}^{(2)}$ and $W_{\bar{z}}^{a(2)}$ are determined from (A.17), (A.18) and (A.19). We can assume the following form

$$
\begin{aligned}
H^{(2)} & =G-i \mathcal{D}_{z}\left(\frac{4}{e^{2}} X^{0} t^{0}+\frac{4}{g^{2}} X^{a} t^{a}\right) H^{\dagger-1}, \\
w_{\bar{z}}^{(2)} t^{0}+W_{\bar{z}}^{a(2)} t^{a} & =i\left(\mathcal{D}_{\bar{z}} G\right) H^{-1}+X^{0} t^{0}+X^{a} t^{a}
\end{aligned}
$$

where $G, X^{0}$ and $X^{a}$ are the fields satisfying

$$
\begin{aligned}
\mathcal{H} & =\mathcal{D}_{\bar{z}}\left[\mathcal{D}_{z}\left(\frac{4}{e^{2}} X^{0} t^{0}+\frac{4}{g^{2}} X^{a} t^{a}\right) H^{\dagger-1}\right]-\left(X^{0} t^{0}+X^{a} t^{a}\right) H, \\
\mathcal{W}_{e}+\mathcal{W}_{g} & =-\mathcal{D}_{z}\left(\mathcal{D}_{\bar{z}} G H^{-1}\right)+\frac{e^{2}}{2} \operatorname{Tr}\left(G H^{\dagger} t^{0}\right) t^{0}+\frac{g^{2}}{2} \operatorname{Tr}\left(G H^{\dagger} t^{a}\right) t^{a} .
\end{aligned}
$$

If we focus on their asymptotic forms, the background fields can be replaced with their vacuum expectation values. Therefore, the equations (A.28) and (A.27) reduces to

$$
\begin{aligned}
\ddot{\phi}^{i} \frac{\partial}{\partial \phi^{i}} \Psi+\dot{\phi}^{i} \dot{\phi}^{j} \frac{\partial}{\partial \phi^{i}} \frac{\partial}{\partial \phi^{j}} \Psi & =-\bar{\partial} \partial\left(\frac{4}{m_{e}^{2}} X^{0} t^{0}+\frac{4}{m_{g}^{2}} X^{a} t^{a}\right)+\left(X^{0} t^{0}+X^{a} t^{a}\right), \\
v \dot{\phi}^{i} \dot{\bar{\phi}}^{j} \frac{\partial}{\partial \phi^{i}} \frac{\partial}{\partial \bar{\phi}^{j}} \Psi & =-4 \partial \bar{\partial} G+2 m_{e}^{2} \operatorname{Tr}\left(G t^{0}\right) t^{0}+2 m_{g}^{2} \operatorname{Tr}\left(G t^{a}\right) t^{a} .
\end{aligned}
$$


These equations can be solved as

$$
\begin{aligned}
G= & -\frac{v}{4}\left(\bar{z}-\bar{z}_{0}\right) \dot{z}_{0} \dot{\bar{z}}_{0} \bar{\partial} \Psi-\frac{v}{m_{g}^{2}}\left(z-z_{0}\right) \dot{\beta}^{i} \dot{\bar{\beta}}^{j} \frac{\partial^{2}}{\partial \beta^{i} \partial \bar{\beta}^{j}} \partial \Psi \\
& +\frac{v}{4}\left[\left(\bar{z}-\bar{z}_{0}\right) \dot{z}_{0} \dot{\bar{\beta}}^{i} \frac{\partial}{\partial \bar{\beta}^{i}}+\left(z-z_{0}\right) \dot{\bar{z}}_{0} \dot{\beta}^{i} \frac{\partial}{\partial \beta^{i}}\right] \Psi \\
X^{0} t^{0}+X^{a} t^{a}= & +\frac{i}{4}\left(\bar{z}-\bar{z}_{0}\right)\left(\ddot{z}_{0}-\dot{z}_{0} \dot{z}_{0} \partial\right) \bar{\partial} \Psi \\
& +\frac{i}{2}\left(\bar{z}-\bar{z}_{0}\right) \dot{z}_{0} \dot{\beta}^{i} \frac{\partial}{\partial \beta^{i}} \bar{\partial} \Psi-\frac{i}{4}\left(z-z_{0}\right) \nabla_{t} \dot{\beta}^{i} \frac{\partial}{\partial \beta^{i}} \Psi .
\end{aligned}
$$

Then, we obtain a solution for the second order fluctuations as

$$
\begin{aligned}
H^{(2)}= & \frac{v}{4}\left(\bar{z}-\bar{z}_{0}\right)\left[\ddot{z}_{0}-\dot{z}_{0}\left(\dot{z}_{0} \partial+\dot{\bar{z}}_{0} \bar{\partial}\right)\right] \Psi \\
+ & \frac{v}{4}\left[\left(\bar{z}-\bar{z}_{0}\right) \dot{z}_{0} \dot{\bar{\beta}}^{i} \frac{\partial}{\partial \bar{\beta}^{i}}+\left(z-z_{0}\right) \dot{\bar{z}}_{0} \dot{\beta}^{i} \frac{\partial}{\partial \beta^{i}}\right] \Psi \\
+ & \frac{v}{2}\left(\bar{z}-\bar{z}_{0}\right) \dot{z}_{0} \dot{\beta}^{i} \frac{\partial}{\partial \beta^{i}} \Psi-\frac{v}{m_{g}^{2}}\left(z-z_{0}\right) \dot{\beta}^{i} \dot{\bar{\beta}}^{j} \frac{\partial^{2}}{\partial \beta^{i} \partial \bar{\beta} j} \partial \Psi \\
& -\frac{v}{m_{g}^{2}} \nabla_{t} \dot{\beta}^{i} \frac{\partial}{\partial \beta^{i}} \partial\left[\left(z-z_{0}\right) \Psi\right]+\{\text { zero modes }\} \\
w_{\bar{z}}^{(2)} t^{0}+W_{\bar{z}}^{a(2)} t^{a}=\quad & \frac{i}{4}\left[\ddot{z}_{0}-\dot{z}_{0}\left(\dot{z}_{0} \partial+\dot{\bar{z}}_{0} \bar{\partial}\right)\right]\left[\left(\bar{z}-\bar{z}_{0}\right) \bar{\partial} \Psi\right] \\
+ & \frac{i}{4}\left[\dot{z}_{0} \dot{\bar{\beta}}^{i} \frac{\partial}{\partial \bar{\beta}^{i}}+\dot{\bar{z}}_{0} \dot{\beta}^{i} \frac{\partial}{\partial \beta^{i}}\right] \bar{\partial}\left[\left(\bar{z}-\bar{z}_{0}\right) \Psi\right] \\
+ & \frac{i}{2}\left(\bar{z}-\bar{z}_{0}\right) \dot{z}_{0} \dot{\beta}^{i} \frac{\partial}{\partial \beta^{i}} \bar{\partial} \Psi-\frac{i}{4}\left(z-z_{0}\right)\left(\dot{\beta}^{i} \dot{\bar{\beta}}^{j} \frac{\partial^{2}}{\partial \beta^{i} \partial \bar{\beta}^{j}}+\nabla_{t} \dot{\beta}^{i} \frac{\partial}{\partial \beta^{i}}\right) \Psi \\
+ & \{\text { zero modes }\} .
\end{aligned}
$$

We can show that these solutions are related to (5.24) and (5.25) by the gauge transformation (A.20) with

$$
\begin{aligned}
A^{(2)}= & {\left[\frac{i}{8}\left(\bar{z}-\bar{z}_{0}\right)\left[\ddot{z}_{0}-\dot{z}_{0}\left(\dot{z}_{0} \partial+\dot{\bar{z}}_{0} \bar{\partial}\right)\right] \Psi+\frac{i}{4}\left(\bar{z}-\bar{z}_{0}\right) \dot{z}_{0} \dot{\beta}^{i} \frac{\partial}{\partial \beta^{i}} \Psi\right.} \\
& \left.-\frac{i c_{g}}{2 m_{g}^{2}} \nabla_{t} \dot{\beta}^{i} \frac{\partial}{\partial \beta^{i}} \partial\left[\left(z-z_{0}\right) \Psi\right]\right]+(\text { h.c. }) .
\end{aligned}
$$

\section{References}

[1] A. A. Abrikosov, "On the Magnetic properties of superconductors of the second group," Sov. Phys. JETP 5, 1174 (1957) [Zh. Eksp. Teor. Fiz. 32, 1442 (1957)]; H. B. Nielsen and P. Olesen, "Vortex-line models for dual strings," Nucl. Phys. B 61, 45 (1973). 
[2] G. 't Hooft, "Magnetic Monopoles In Unified Gauge Theories," Nucl. Phys. B 79, 276 (1974); A. M. Polyakov, "Particle spectrum in quantum field theory," JETP Lett. 20, 194 (1974) [Pisma Zh. Eksp. Teor. Fiz. 20, 430 (1974)].

[3] A. A. Belavin, A. M. Polyakov, A. S. Shvarts and Yu. S. Tyupkin, "Pseudoparticle solutions of the Yang-Mills equations," Phys. Lett. B 59, 85 (1975).

[4] N. S. Manton and P. Sutcliffe, "Topological solitons," Cambridge, UK: Univ. Pr. (2004) 493 $p$

[5] N. S. Manton, "A Remark On The Scattering Of Bps Monopoles," Phys. Lett. B 110 (1982) 54.

[6] M. F. Atiyah and N. J. Hitchin, "Low-Energy Scattering Of Nonabelian Monopoles," Phys. Lett. A 107, 21 (1985).

[7] M. F. Atiyah and N. J. Hitchin, "The Geometry and Dynamics of Magnetic Monopoles. M.B. Porter Lectures," PRINCETON, USA: UNIV. PR. (1988) 133p

[8] G. W. Gibbons and N. S. Manton, "The Moduli space metric for well separated BPS monopoles," Phys. Lett. B 356, 32 (1995) arXiv:hep-th/9506052.

[9] K. M. Lee, E. J. Weinberg and P. Yi, "The Moduli Space of Many BPS Monopoles for Arbitrary Gauge Groups," Phys. Rev. D 54, 1633 (1996) arXiv:hep-th/9602167.

[10] C. H. Taubes, "Arbitrary N: Vortex Solutions To The First Order Landau-Ginzburg Equations," Commun. Math. Phys. 72, 277 (1980).

[11] T. M. Samols, "Vortex Scattering," Commun. Math. Phys. 145, 149 (1992).

[12] H. Y. Chen and N. S. Manton, "The Kaehler potential of Abelian Higgs vortices," J. Math. Phys. 46, 052305 (2005) arXiv:hep-th/0407011.

[13] N. S. Manton and J. M. Speight, "Asymptotic interactions of critically coupled vortices," Commun. Math. Phys. 236, 535 (2003) arXiv:hep-th/0205307.

[14] S. Krusch and J. M. Speight, "Exact moduli space metrics for hyperbolic vortices," arXiv:0906.2007 [hep-th].

[15] E. Witten, "Some exact multipseudoparticle solutions of classical Yang-Mills theory," Phys. Rev. Lett. 38, 121 (1977).

[16] A. Hanany and D. Tong, "Vortices, instantons and branes," JHEP 0307, 037 (2003) arXiv:hep-th/0306150.

[17] R. Auzzi, S. Bolognesi, J. Evslin, K. Konishi and A. Yung, "Nonabelian superconductors: Vortices and confinement in N = 2 SQCD," Nucl. Phys. B 673, 187 (2003) arXiv:hep-th/0307287. 
[18] D. Tong, "TASI lectures on solitons," arXiv:hep-th/0509216; "Quantum Vortex Strings: A Review," Annals Phys. 324, 30 (2009) [arXiv:0809.5060 [hep-th]]; K. Konishi, "The magnetic monopoles seventy-five years later," Lect. Notes Phys. 737, 471 (2008) arXiv:hep-th/0702102]; "Advent of Non-Abelian Vortices and Monopoles- further thoughts about duality and confinement," Prog. Theor. Phys. Suppl. 177, 83 (2009) arXiv:0809.1370 [hep-th]]; M. Shifman and A. Yung, "Supersymmetric Solitons and How They Help Us Understand Non-Abelian Gauge Theories," Rev. Mod. Phys. 79, 1139 (2007) arXiv:hep-th/0703267]; an expanded version in Cambridge University Press, 2009.

[19] M. Eto, Y. Isozumi, M. Nitta, K. Ohashi and N. Sakai, "Solitons in the Higgs phase: The moduli matrix approach," J. Phys. A 39 (2006) R315 arXiv:hep-th/0602170.

[20] M. Shifman and A. Yung, "Non-Abelian string junctions as confined monopoles," Phys. Rev. D 70, 045004 (2004) arXiv:hep-th/0403149 ; A. Hanany and D. Tong, "Vortex strings and four-dimensional gauge dynamics," JHEP 0404, 066 (2004) arXiv:hep-th/0403158.

[21] Y. Isozumi, M. Nitta, K. Ohashi and N. Sakai, "All exact solutions of a 1/4 Bogomol'nyiPrasad-Sommerfield equation," Phys. Rev. D 71, 065018 (2005) arXiv:hep-th/0405129.

[22] M. Eto, Y. Isozumi, M. Nitta, K. Ohashi and N. Sakai, "Instantons in the Higgs phase," Phys. Rev. D 72, 025011 (2005) arXiv:hep-th/0412048.

[23] M. Eto, Y. Isozumi, M. Nitta and K. Ohashi, "1/2, 1/4 and 1/8 BPS equations in SUSY Yang-Mills-Higgs systems: Field theoretical brane configurations," Nucl. Phys. B 752, 140 (2006) arXiv:hep-th/0506257.

[24] M. Eto, Y. Isozumi, M. Nitta, K. Ohashi and N. Sakai, "Moduli space of non-Abelian vortices," Phys. Rev. Lett. 96 (2006) 161601 arXiv:hep-th/0511088.

[25] M. Eto, Y. Isozumi, M. Nitta, K. Ohashi and N. Sakai, "Manifestly supersymmetric effective Lagrangians on BPS solitons," Phys. Rev. D 73, 125008 (2006) arXiv:hep-th/0602289.

[26] M. Eto, K. Konishi, G. Marmorini, M. Nitta, K. Ohashi, W. Vinci and N. Yokoi, "Non-Abelian vortices of higher winding numbers," Phys. Rev. D 74 (2006) 065021 arXiv:hep-th/0607070.

[27] M. Eto, K. Hashimoto, G. Marmorini, M. Nitta, K. Ohashi and W. Vinci, "Universal reconnection of non-Abelian cosmic strings," Phys. Rev. Lett. 98 (2007) 091602 arXiv:hep-th/0609214.

[28] M. Eto et al., "Non-Abelian duality from vortex moduli: a dual model of color-confinement," Nucl. Phys. B 780, 161 (2007) arXiv:hep-th/0611313.

[29] M. Eto et al., "On the moduli space of semilocal strings and lumps," Phys. Rev. D 76 (2007) 105002 [arXiv:0704.2218 [hep-th]]. 
[30] M. Eto, T. Fujimori, T. Nagashima, M. Nitta, K. Ohashi and N. Sakai, "Multiple Layer Structure of Non-Abelian Vortex," Phys. Lett. B 678, 254 (2009) arXiv:0903.1518 [hepth]].

[31] M. Eto, T. Fujimori, G. Marmorini, M. Nitta, K. Ohashi and N. Sakai, in preparation.

[32] T. Vachaspati and A. Achucarro, "Semilocal cosmic strings," Phys. Rev. D 44, 3067 (1991); A. Achucarro and T. Vachaspati, "Semilocal and electroweak strings," Phys. Rept. 327, 347 (2000) [Phys. Rept. 327, 427 (2000)] arXiv:hep-ph/9904229].

[33] M. Shifman and A. Yung, "Non-Abelian semilocal strings in N = 2 supersymmetric QCD," Phys. Rev. D 73, 125012 (2006) arXiv:hep-th/0603134].

[34] M. Eto, T. Fujimori, S. B. Gudnason, K. Konishi, M. Nitta, K. Ohashi and W. Vinci, "Constructing Non-Abelian Vortices with Arbitrary Gauge Groups," Phys. Lett. B 669, 98 (2008) arXiv:0802.1020 [hep-th]].

[35] L. Ferretti, S. B. Gudnason and K. Konishi, "Non-Abelian vortices and monopoles in SO(N) theories," Nucl. Phys. B 789, 84 (2008) [arXiv:0706.3854 [hep-th]].

[36] M. Eto, T. Fujimori, S. B. Gudnason, M. Nitta and K. Ohashi, "SO and USp Káhler and Hyper-Káhler Quotients and Lumps," Nucl. Phys. B 815, 495 (2009) arXiv:0809.2014 [hepth]].

[37] M. Eto et al., "Non-Abelian Vortices in $\mathrm{SO}(\mathrm{N})$ and USp(N) Gauge Theories," JHEP 0906, 004 (2009) [arXiv:0903.4471 [hep-th]].

[38] S. B. Gudnason and K. Konishi, "Low-energy U(1) x USp(2M) gauge theory from simple high-energy gauge group," arXiv:1002.0850 [hep-th].

[39] J. M. Speight, "Static intervortex forces," Phys. Rev. D 55, 3830 (1997) arXiv:hep-th/9603155.

[40] M. Eto, T. Fujimori, T. Nagashima, M. Nitta, K. Ohashi and N. Sakai, "Dynamics of Strings between Walls," Phys. Rev. D 79, 045015 (2009) [arXiv:0810.3495 [hep-th]].

[41] R. S. Ward, "Slowly Moving Lumps In The Cp**1 Model In (2+1)-Dimensions," Phys. Lett. B 158, 424 (1985).

[42] R. A. Leese and T. M. Samols, "Interaction of semilocal vortices," Nucl. Phys. B 396, 639 (1993).

[43] M. Eto, T. Fujimori, Y. Isozumi, M. Nitta, K. Ohashi, K. Ohta and N. Sakai, "Non-Abelian vortices on cylinder: Duality between vortices and walls," Phys. Rev. D 73, 085008 (2006) arXiv:hep-th/0601181.

[44] M. Eto, T. Fujimori, M. Nitta, K. Ohashi, K. Ohta and N. Sakai, "Statistical Mechanics of Vortices from D-branes and T-duality," Nucl. Phys. B 788, 120 (2008) arXiv:hep-th/0703197]. 
[45] J. M. Baptista, "Non-abelian vortices on compact Riemann surfaces," Commun. Math. Phys. 291, 799 (2009) arXiv:0810.3220 [hep-th]]; A. D. Popov, "Integrability of Vortex Equations on Riemann Surfaces," Nucl. Phys. B 821, 452 (2009) arXiv:0712.1756 [hep-th]]; "NonAbelian Vortices on Riemann Surfaces: an Integrable Case," Lett. Math. Phys. 84, 139 (2008) arXiv:0801.0808 [hep-th]].

[46] N. S. Manton and N. Sakai, "Maximally Non-Abelian Vortices from Self-dual Yang-Mills Fields," arXiv:1001.5236 [hep-th].

[47] B. Collie and D. Tong, "The Dynamics of Chern-Simons Vortices," Phys. Rev. D 78, 065013 (2008) [arXiv:0805.0602 [hep-th]]; B. Collie, "Dyonic Non-Abelian Vortices," J. Phys. A 42, 085404 (2009) arXiv:0809.0394 [hep-th]]; S. B. Gudnason, "Non-Abelian Chern-Simons vortices with generic gauge groups," Nucl. Phys. B 821, 151 (2009) arXiv:0906.0021 [hepth]].

[48] R. Auzzi, M. Eto and W. Vinci, "Static Interactions of non-Abelian Vortices," JHEP 0802, 100 (2008) arXiv:0711.0116 [hep-th]]; R. Auzzi, M. Eto, S. B. Gudnason, K. Konishi and W. Vinci, "On the Stability of Non-Abelian Semi-local Vortices," Nucl. Phys. B 813, 484 (2009) arXiv:0810.5679 [hep-th]]. 